\title{
Catálogo de Apoidea da região neotropical (Hymenoptera, Colletidae). III. Colletini ${ }^{1}$
}

\author{
Jesus Santiago Moure ${ }^{2}$ \\ Danuncia Urban ${ }^{2}$
}

\begin{abstract}
Catalogue of the Apoidea of the Neotropical region (Hymenoptera, Colletidae). III. Colletini. The type locality, depositary museums, geographical occurrences, biological behaviour, flowers visited and taxonomical notes from the species of the neotropical Colletini are mentioned. The following nomenclatural changes are introduced: Monidia Cockerell, 1905; Rhynchocolletes Moure, 1943; Hemicotelles Toro \& Cabezas, 1997; Xanthocotelles Toro \& Cabezas, 1978 and Colletes griseus Smith, 1879 are revalidated; Colletes desantisi nom. n. is proposed to Colletes similis Joergensen, 1912, non Schenck, 1853 nec Robertson, 1904; a new combination to Mourecotelles biciliatus (Cockerell, 1918) is given. Lectotypes of the following species are designated: Colletes araucariae Friese, 1910; C. argentinus Friese, 1908; C. atripilis Vachal, 1909; C. azureus Friese, 1912; C. chalybaeus Friese, 1910; C. ciliatus Friese, 1910; C. clarus Joergensen, 1912; C. cognata Spinola, 1851; C. costaricensis Friese, 1916; C. cyaniventris Spinola, 1851; C. dimidiata Spinola, 1851; C. enodis Vachal, 1909; C. eupogonites Moure, 1949; C. extensicornis Vachal, 1909; C. fulvipes Spinola, 1851; C. glycyrrhizae Joergensen, 1912; C. griseus Smith, 1879; C. joergenseni Friese, 1910; C. laticeps Friese, 1910; C. longipes Friese, 1910; C. lycii Joergensen, 1912; C. mininca Cockerell, 1914; C. motaguensis Cockerell, 1912; C. murinus Friese, 1900; C. musculus Friese, 1910; C. neuqueenensis Friese, 1910; C. perplexus Smith, 1879; C. peruvicus Cockerell, 1913; C. plantaris Vachal, 1909; C. senilis Smith, 1879; C. speculiventris Cockerell, 1917; C. pinnatus Vachal, 1909; C. rugicollis Friese, 1900; C. schrottkyi Joergensen, 1912; C. sicheli Vachal, 1909; C. spiloptera Cockerell, 1917; C. steinbachi Friese, 1910; C. sulcatus Vachal, 1909; C. tingoensis Cockerell, 1926; C. tomentosus Friese, 1910; C. vachali Joergensen, 1912; C. virgatus Vachal, 1904; C. weiskei Friese, 1912; Monia grisea Westwood, 1875. Are recognized as new synonyms: Colletes argentinus Friese, 1908, syn. n. of C. rugicollis Friese, 1900; C. campoi Herbst, 1920, syn. n. of C. rhodaspis Cockerell, 1909; C. viridans Vachal, 1909, syn. n. of C. cyanescens (Haliday, 1837).

KEY WORDS. Hymenoptera, Apoidea, Colletidae, Colletini, Neotropical, catalogue
\end{abstract}

MiCHENER (1944) definiu os Colletini como similares aos Paracolletini porém com a porção basal do propódeo curta e provida com carenas longitudinais freqüentemente separando uma série de fóveas; e, aparentemente contendo só o gênero Colletes Latreille, 1802; TORO \& CABEZAS (1977) deram a conhecer mais dois gêneros Hemicotelles e Mourecotelles, e estes mesmos autores em 1978, publicaram Xanthocotelles, mais um Colletini neotropical. MICHENER (1989) propôs uma chave para as tribos americanas de Colletinae, deixando em sinonímia de Mourecotelles tanto Hemicotelles como Xanthocotelles e considerando Monidia Cockerell, 1905, na sinonímia de Colletes. MichenER (2000), tratou Colletinae como subfamília, sem subdivisão em tribos.

1) Contribuição número 1276 do Departamento de Zoologia, Universidade Federal do Paraná.

2) Departamento de Zoologia, Universidade Federal do Paraná. Caixa Postal 19020, 81531-990 Curitiba, Paraná, Brasil. Bolsista do CNPq. 
Neste catálogo é mantida a tribo Colletini. As informações bibliográficas mais frequentes estão listadas nas "Referências Bibliográficas", são dadas abreviadamente quando repetidas na mesma espécie; as abreviações latinas são as comumente encontradas em trabalhos de taxonomia e as procedências novas foram obtidas junto aos autores consultados.

Os acrônimos dos museus citados no texto são relacionados a seguir: (AMNH) American Museum of Natural History, New York, Estados Unidos; (ANSP) Academy of Natural Sciences, Philadelphia, Estados Unidos; (BMNH) The Natural History Museum, London, Inglaterra; (CAS) California Academy of Sciences, San Francisco, Estados Unidos; (DZUP) Coleção de Entomologia Pe. J.S. Moure, Departamento de Zoologia da Universidade Federal do Paraná, Curitiba, Brasil; (IML) Instituto Miguel Lillo, Universidad Nacional de Tucumán, San Miguel de Tucumán, Argentina; (MLP) Facultad de Ciencias Naturales y Museo, Universidad Nacional de La Plata, La Plata, Argentina; (MBR) Museo Argentino de Ciencias Naturales "Bernardino Rivadavia", Buenos Aires, Argentina; (MCZ) Museum of Comparative Zoology, Harvard University, Cambridge, Estados Unidos; (MIZT) Museo ed Istituto di Zoologia Sistematica, Universitá di Torino, Torino, Itália; (MNHP) Muséum National d'Histoire Naturelle, Paris, França; (MHNS) Museu Nacional de Historia Natural, Santiago, Chile; (MNHU) Museum für Naturkunde der Humboldt-Universität zu Berlin, Alemanha; (MZSP) Museu de Zoologia, Universidade de São Paulo, Brasil (ex Departamento de Zoologia da Secretaria de Agricultura); (OSUC) Oregon State University, Corvallis, Oregon, Estados Unidos; (SEMK) Snow Entomological Museum, University of Kansas, Lawrence, Estados Unidos; (UNL) University of Nebraska, Lincoln, Estados Unidos; (USNM) National Museum of Natural History, Washington, Estados Unidos. A Coleção Schrottky foi depositada pelo autor em locais diversos: MBR, MZSP, Instituto Oswaldo Cruz, MNHU, e no Paraguai, em local não conhecido; e a Coleção Toro se encontra em Valparaíso, Chile.

\section{CONTEÚDO}

Introdução........................................... 1

Mourecotelles

Colletes

Rhynchocolletes.

24

Hemicotelles ...................................... 22

Monidia

Xanthocotelles

Índice

\section{Colletes Latreille, 1802}

Colletes Latreille, 1802. Histoire Naturelle des Fourmis: 423. - 1802. Histoire Naturelle Générale et Particulière des Crustacés et des Insectes 3: 372. - Michener, 1989. Univ. Kansas Sci. Bull. 53 (11): 685; syn.: Monia Westwood, 1875; Monidia Cockerell, 1905; Rhynchocolletes Moure, 1943. Espécie-tipo: Apis succincta Linnaeus, 1758. Monotípico.

Colletes foi proposto por Latreille em 1802, em duas publicações, nas duas Latreille indicou Apis succincta Linnaeus, 1758, como espécie-tipo "Exemple du genre", sem fazer a combinação como espécie de Colletes. O nome Colletes é de origem grega, e tem sido usado indiferentemente como masculino ou como feminino. Foi definido como gênero masculino no Bull. Zool. Nomenclature 50 (1): 85, Opinion 1713 publicado em 1993. 
Taxonomia. Spinola, 1851: 217 (redescreveu o gênero). - Schrottky, 1902, Rev. Mus. Paulista. 5: 343-344 (caracteres diagnósticos e chave para espécies brasileiras). - Schrottky, 1907. An. Ci. Paraguayos 7: 4 (caracteres e distribuição geográfica; chave para as espécies do Paraguai). - Vachal, 1909. Rev. d' Ent., Caen, 28: 54-56 (chave para espécies do Chile). - Friese, 1910. Zool. Jahrb. Syst. 29: 653-654 (chave para as espécies). - Metz, 1910. Pomona C. Jour. Ent. 2: 191-208 (espécies novas de Colletes do México). - Joergensen, 1912. Zool. Jahrb. Syst. 32: 99-100 (chave para as espécies de Mendoza). - Friese, 1912. Deutsch. ent. Ztschr. p. 366-367 (chave para as espécies). - Richards, 1935. Tr. Royal Ent. Soc. London 83: 169 (examinou o tipo de Apis succincta, uma fêmea, na Coleção da Linnean Society, Burlington House). - Michener, 1997. Sci. Papers, Nat. Hist. Mus. Univ. Kansas 1: 16 (considerou Colletes e Evodia Panzer, 1806, isotípicos por sinonímia). - Michener, 2000. The Bees of the World: 135 (conservou Monidia Cockerell e Rhynchocolletes Moure na sinonímia de Colletes).

Biologia. Spinola, 1851: 217 (referiu-se a ninhos em lugares expostos ao sol; consistindo de vários tubos cilíndricos). - Schrottky, 1907: 4, 5 (repetiu os dados de Spinola, 1851, sobre os ninhos). - Claude-Joseph, 1926. Ann. Sci. Nat., Zool., Paris, (10) 9: 120-121 (estudando espécies de Colletes do Chile teceu comentários sobre as galerias dos ninhos, cilíndricas e tortuosas, frequentemente ramificadas, pouco profundas e as células membranosas provisionadas com uma pasta com mel, muito fluida). - Ruiz, 1944. Rev. Chilena Hist. Nat. 48: 202 (comentou presença de parasitas em ninhos: Epeolus Latreille, 1802, Coelioxys Latreille, 1809 e Diptera).

aethiops Cresson. PANAMÁ, Chiriqui (localidade-tipo), El Volcán. MÉXICO, Orizaba.

Colletes aethiops Cresson, 1868. Proc. Boston Soc. Nat. Hist. 12: 169. Síntipo fêmea na ANSP.

Taxonomia. Cockerell, 1899. Cat. Abejas de México p. 4 (ocorrência). Cresson, 1916. Mem. Amer. Ent. Soc. 1: 106 (comentou a boa conservação do tipo). - Michener, 1954. Bull. Amer. Mus. Nat. Hist. 104 (1): 16-17 (redescrição).

alocochila Moure. CHILE, Antofagasta, San Pedro de Atacama (localidade-tipo); Tarapacá, Arica, Putre $(3650 \mathrm{~m})$.

Colletes alocochila Moure, 1956. Dusenia 7 (4): 208. Holótipo fêmea, alótipo macho e dois parátipos no DZUP.

antiguensis Cockerell. GuATEMALA, Antigua (localidade-tipo).

Colletes antiguensis Cockerell, 1912. Ann. Mag. Nat. Hist. (8) 9: 565. Síntipo fêmea no AMNH, examinado por J.S. Moure.

Taxonomia. Cockerell, 1912: 565 (comparou com C. niger Swenk, 1904).

araucariae Friese. CHILE, Concepción (localidade-tipo), Rancagua. ARgENTINA.

Colletes araucariae Friese, 1910. Zool. Jahrb. Syst. 29: 649. Lectótipo fêmea no MNHU, coletado em Concepción em 7.III.1908 por P. Herbst, aqui designado; foi examinado por J.S. Moure. 
Taxonomia. Friese, 1910: 649 (comparou com C. tomentosus Friese, 1910). - Friese, 1912: 367 (chave para identificação das espécies).

Biologia. Jaffuel \& Pirión, 1926. Rev. Chilena Hist. Nat. 30: 363 (sobre Adesmia DC.). - Claude-Joseph, 1926. Ann. Sci. Nat., Zool., Paris, (10) 9: 125-127, figs 8, 9 (nidificação na borda dos caminhos, taludes argilosos ou arenosos cobertos de musgo ou grama; cada fêmea cavou uma galeria horizontal ou oblíqua). - Janvier, 1933. Ann. Sci. Nat., Zool, Paris (10) 16: 325 (encontrou ninhos parasitados por Isepeolus luctuosus (Spinola, 1851); observou Isepeolus triseriatus [= Melectoides triseriatus (Friese,1908)] voando sobre as colônias e se reproduzindo em seus ninhos). - Ruiz, 1944. Rev. Chilena Hist. Nat. 48: 204-205 (coletando nectar de Labiatae [= Lamiaceae] e de Compostas [= Asteraceae]). - Stephen, 1954. Univ. Kansas Sci. Bull. 36 (1) 6: 161 (comentou a construção de séries lineares de quatro a oito células de cria).

Morfologia. McGinley, 1981. Univ. Calif. Publ., Entomology 91: 89-90 (descreveu a larva e comparou-a com a de C. thoracicus Smith, 1853).

atacamanus Moure. CHILE, Atacama, San Pedro (localidade-tipo).

Colletes atacamensis Moure, 1956. Dusenia 7 (4): 204; nom. praeoc. Holótipo fêmea, alótipo macho e sete parátipos no DZUP.

Colletes atacamanus Moure, 1997. Revta. Bras. Zool. 14 (2): 513; nom. n. para Colletes atacamensis Moure, 1956 (non Janvier, 1955).

Taxonomia. Moure, 1956: 206 (achou a espécie semelhante a C. peruvica Cockerell, 1913).

atacamensis Janvier. CHILE, Atacama, Quillaga (localidade-tipo).

Colletes atacamensis Janvier, 1955. Ann. Sci. Nat., Zool., Paris, (11) 17:

316. Síntipos machos e fêmeas; provavelmente depositados no MNHP. Biologia. Janvier, 1955: 316-317 (observou nidificação em terra argilosa ressecada pelo sol ardente; duas ou três fêmeas em uma mesma galeria cilíndrica horizontal, a galeria com duas a três ramificações, e galerias secundárias em direções diversas).

atripes Smith. CHILE, Coquimbo (localidade-tipo).

Colletes dimidiata Spinola, 1851, in Gay. Hist. Fis. Pol. Chile, Zool. 6: 225;

nom. praeoc. Lectótipo fêmea no MIZT, aqui designado; foi examinado por J.S. Moure.

Colletes atripes Smith, 1854. Cat. Hym. Br. Mus. 2 p. 418; nom. n. para Colletes dimidiata Spinola (non Brullé, 1840).

Biologia. Jaffuel \& Pirión, 1926. Rev. Chilena Hist. Nat. 30: 364 (visitando Adesmia arborea Bert.).

azteka Cresson. MÉxICO (localidade-tipo), Orizaba.

Colletes azteka Cresson, 1868. Proc. Boston Soc. Nat. Hist. 12: 169. Síntipo

fêmea na ANSP, examinado por J.S. Moure. 
Colletes azteca [sic]; Cockerell, 1899. Cat. Abejas de México p. 4.

Taxonomia. Cresson, 1916. Mem. Amer. Ent. Soc. 1: 106 (selecionou uma fêmea com o n² 2153 , como tipo).

azureus Friese. ARGENTINA, Neuquen (localidade-tipo), Cordilheira.

Colletes azureus Friese, 1912. Deutsch. ent. Ztschr. p. 365. Lectótipo fêmea no MNHU, coletado em XI. 1910, aqui designado; foi examinado por J.S. Moure.

Taxonomia. Friese, 1912: 365 (comparou com C. chalybaeus Friese, 1910).

bicolor Smith. ARgentina, Mendoza (localidade-tipo) Potrerillos, San Ignacio, Salta, Neuquen, Chubut, Patagonia. CHILE, Concepción, Osorno, Talca, Valle de Choapa, Punta de Lobos.

Colletes bicolor Smith, 1879. Descr. N. Sp. Hym. Br. Mus. p. 3. Síntipo fêmea provavelmente no BMNH. - Joergensen, 1912. An. Mus. Nac. Buenos Aires 22: 300; syn. Biglossa andina.

Biglossa andina Joergensen, 1909. Deutsch. ent. Ztschr. p. 221. Síntipos macho e fêmea. Depositário desconhecido.

Taxonomia. Schrottky, 1913. An. Soc. Ci. Arg. 75: 236 (ocorrência). Jaffuel \& Pirión, 1926. Rev. Chilena Hist. Nat. 30: 92 (ocorrência no Chile).

Biologia. Jensen-Haarup, 1908, Flora og Fauna 10: 99 (em flores de Physalis L.). - Joergensen, 1909: 221 (ao descreveu B. andina, relacionou as plantas: Baccharis serrulata (Lam.) Person, B. pingrea [sic] var. angustissima $[=$ B. pingraea DC.] e Lycium gracile [= Lycium chilense Bert.] e a ocorrência em Chacras de Coria, Potrerillos e San Ignacio, localidades de Mendoza). - Joergensen, 1912. An. Mus. Nac. Buenos Aires, 22: 300 (observou de novembro a abril sobre Baccharis serrulata, B. salicifolia Person, Tamarix africana Poir., Acasia [sic] furcata [= Acacia furcata Desv.], Prosopis campestris Gris., P. alpataco Phil., Medicago sativa L., Glycyrrhiza astragalina Gill.; Lycium gracile [= Lycium chilense Bert.], Lycium argentinum Hieron, Physalis viscosa L., Solanum elaeagnifolium Cav., S. atriplicifolium Gill. ex Nees; Sphaeralcea bonariensis Griseb). Jaffuel \& Pirión, 1926. Rev. Chilena Hist. Nat. 30: 92 (machos e fêmeas sobre flores de Prosopis alpataco Phil., fêmeas sobre flores de Clematis hilarii [= Clematis denticulata Vell.], Lycium chilense, L. argentinum, Glycyrrhiza astragalina Gill.). - Claude-Joseph, 1926. Ann. Sci. Nat., Zool., Paris, (10) 9: 131-132, fig. 13 (observou que muitos indivíduos se estabeleciam nas vizinhanças para nidificar, cada um fazendo uma galeria com ramificações para o fundo, a última com uma só célula de cria; e exemplares voando sobre Eryngium paniculatum, Eugenia corralensis Phil., Alstroemeria L. e Senecio (Tourn.) L.). - Ruiz, 1944. Rev. Chilena Hist. Nat. 48: 206-207 (notas sobre ninhos e exemplares voando sobre Erygium [sic] paniculatum [= Eryngium paniculatum Cav. \& Dombay], Alstroemeria e Senecio). 
bombiformis Metz. MÉXICO, Guadalajara (localidade-tipo).

Colletes bombiformis Metz, 1910. Pomona C. Jour. Ent. 2: 206. Holótipo fêmea depositado na OSUC.

Taxonomia. Metz, 1910: 207 (comentou que poderia ser C. aethiops Cresson, 1868 , com diferenças na pilosidade).

brethesi Joergensen. ARgentina, Mendoza, Chacras de Coria (localidade-tipo).

Colletes brethesi Joergensen, 1912. Zool. Jahrb. Syst. 32: 94. Holótipo fêmea no MLP.

Taxonomia. Joergensen, 1912: 94 (comparou com C. cyaneus Holmberg, 1903). - Joergensen, 1912. An. Mus. Nac. Buenos Aires, 22: 300 (comentou a semelhança de $C$. brethesi com C. virgatus Vachal, 1904). - Durante \& Díaz, 1998. Rev. Mus. La Plata (N. S.) (33): 8 (relacionaram na listagem dos tipos de Apoidea do MLP).

brevinodis Vachal. BoLíviA (localidade-tipo).

Colletes brevinodis Vachal, 1909. Rev. d'Ent., Caen, 28: 52. Holótipo macho no MNHP, examinado por J.S. Moure.

Taxonomia. Vachal, 1909: 52 (comparou com C. rutilans Vachal, 1909).

bruneri Swenk. CosTA RICA, Monte Redondo (localidade-tipo), 4.000 pés.

Colletes bruneri Swenk, 1904. Can. Ent. 36: 77. Holótipo fêmea na UNL. capitatus Metz. MÉXICO, Guadalajara (localidade-tipo).

Colletes capitata Metz, 1910. Pomona C. Jour. Ent. 2: 204. Quatro síntipos fêmeas depositados na OSUC.

Taxonomia. Friese, 1910. Zool. Jahrb. Syst. 29: 646 (comparou com C. virgatus Vachal, 1904 e C. cyaneus Holmberg, 1903).

chalybaeus Friese. ArgentinA, La Plata, Buenos Aires (localidade-tipo), Neuquén. Colletes chalybaeus Friese, 1910. Zool. Jahrb. Syst. 29: 646. Lectótipo fêmea no MNHU, coletado em 15. XII. 05, aqui designado; um paralectótipo fêmea de Neuquén no USNM; examinados por J.S. Moure.

Taxonomia. Friese, 1910: 646 (comparou com C. virgatus Vachal, 1904, e C. cyaneus Holmberg, 1903).

chubutensis Cockerell. ARgEnTINA, Chubut (localidade-tipo), Patagonia Colletes chubutensis Cockerell, 1918. Can. Ent. 50: 137. Síntipo macho no USNM, examinado por J.S. Moure.

Taxonomia. Cockerell, 1918: 138 (Comentou que na chave de Friese [1910] sairia próxima de C. biciliatus Cockerell, 1918 [= Mourecotelles biciliatus (Cockerell, 1918)]).

clarus Joergensen. ArgentinA, Mendoza, Chacras de Coria (localidade-tipo). Colletes clarus Joergensen, 1912. Zool. Jahrb. Syst. 32: 98. Lectótipo macho no MLP, paralectótipo fêmea no MLP, aqui designados. 
Taxonomia. Durante \& Díaz, 1998. Rev. Mus. La Plata (N. S.) (33): 8 (examinaram os síntipos macho e fêmea, o macho sem o tagma cefálico e rotulado com etiqueta de lectótipo por Moure \& Ogloblin, os quais não publicaram a designação).

Biologia. Joergensen, 1912. An. Mus. Nac. Buenos Aires 22: 301 (sobre flores de Patagonium gilliesii [sic] [= Adesmia giliesii Hook. \& Arn.]).

clematidis Joergensen. ARGENTINA, Mendoza, Chacras de Coria (localidade-tipo), San Ignacio; Neuquen.

Colletes clematidis Joergensen, 1912. Zool. Jahrb. Syst. 32: 93. Três síntipos, dois machos e uma fêmea, no MLP.

Taxonomia. Durante \& Díaz, 1998. Rev. Mus. La Plata (N.S.) (33): 8 (examinaram o material-tipo no MLP).

Biologia. Joergensen, 1912: 93 (observou exemplares visitando flores de Clematis hilarii Spreng. [= C. denticulata Vell.], Prosopis alpataco Phil., Prosopis campestris Gris., Glycorrhyza [sic] astragalina [= Glycyrrhiza astragalina Gill]).

cognatus Spinola. CHILE (localidade-tipo), Concepción. ARGENTINA.

Colletes cognata Spinola, 1851, in Gay. Hist. Fis. Pol. Chile, Zool. 6: 223.

Lectótipo macho no MIZT, aqui designado; foi examinado por J.S. Moure.

Taxonomia. Spinola, 1851: 223-224 (comparou C. cognata com C. succincta e com C. marginita [sic] nom. praeoc. descrita no mesmo trabalho [= Lonchopria zonalis (Reed, 1892), um Paracolletini]).

costaricensis Friese. CosTA RICA, San José (localidade-tipo), San Mateo. EQUADOR, Sta. Inez. ColÔMBIA, Popayan. BolíviA, Cordilheira Ocidental.

Colletes costaricensis Friese, 1916. Stett. Ent. Ztg. 77: 302. Lectótipo fêmea no MNHU, aqui designado; um paralectótipo fêmea no USNM; foram examinados por J.S. Moure.

Taxonomia. Friese, 1916: 302 (comparou com C. daviesanus Smith, 1846 [espécie paleártica] e $C$. cognata Spinola).

cyanescens (Haliday). CHILE (localidade-tipo), Províncias centrais do CHILE (localidade-tipo de C. cyaniventris); Quillota, Niuble [sic] [= Ñuble], Concepción. CHILE (localidade-tipo de C. atripilis e de C. viridans)

Andrena cyanescens Haliday, 1837. Tr. Linn. Soc. London, 17 (3): 321. Tipo não examinado.

Colletes cyani-ventris [sic] Spinola, 1851, in Gay. Hist. Fis. Pol. Chile, Zool. 6: 224. Lectótipo fêmea no MIZT, aqui designado; foi examinado por J.S. Moure.

Colletes cyanescens; Cockerell, 1904. Ent. News 15: 277; syn.: C. cyaniventris. - Cockerell, 1917. Ann. Mag. Nat. Hist. (8) 19: 478; syn. C. atripilis. 
Colletes viridans Vachal, 1909. Rev. d' Ent., Caen, 28: 55, 56. Um síntipo fêmea no MNHP, examinado por J.S. Moure. Syn. n.

Colletes atripilis Vachal, 1909. Rev. d' Ent., Caen, 28: 55. Lectótipo macho no MNHP, aqui designado, paralectótipo fêmea na mesma coleção; foram examinados por J.S. Moure.

Taxonomia. Vachal, 1909: 55, 56 (colocou Colletes viridans na chave para as espécies com abdome metálico que ocorrem no Chile, não descreveu os exemplares; citou localidades do Chile).

Biologia. Jaffuel \& Pirión, 1926. Rev. Chilena Hist. Nat. 30: 364 (observaram exemplares visitando flores de Escallonia illinita Presl., onde notaram o mimetismo entre esta abelha e o díptero Volucella azurea $[=$ Copestylum azureum (Philippi, 1865)] que também visita a mesma planta). - Claude-Joseph, 1926. Ann. Sci. Nat., Zool., Paris, (10) 9: 128-130, figs 6, 7, 11 (ninhos superficiais ou galerias profundas no solo; sobre flores de Quillaja saponaria, Deutzia crenata Sieb. \& Zucc. e Baccharis L.). - Janvier, 1933. Ann. Sci. Nat., Zool, Paris (10) 16: 325 (referiu-se a ninhos parasitados por Isepeolus luctuosus (Spinola, 1851) e observou Epeoloides septemnotatus [= Isepeolus septemnotatus (Spinola,1851)], e Isepeolus triseriatus [= Melectoides triseriatus (Friese,1908)] voando sobre as colônias e se reproduzindo em seus ninhos). - Ruiz, 1944. Rev. Chilena Hist. Nat. 48: 211 (ninhos no interior dos bosques com cinco ou mais células em cada galeria e machos em enxames sobre os arbustos ou árvores próximos à colônia; visitam flores de Quillaja saponaria Molina, Colletia ulcina[sic] [= Colletia ulicina Gill. \& Hook.], Colletia sp., Baccharis rosmarinifolia Kook. \& Arn.).

cyaneus Holmberg. ARgentina, Buenos Aires, Las Conchas (localidade-tipo).

Colletes cyaneus Holmberg, 1903. An. Mus. Nac. Buenos Aires II (3): 468. Síntipos: duas fêmeas coletadas, respectivamente em II. [18]79 e III. [18]80, provavelmene depositadas no MBR.

Taxonomia. Schrottky, 1907. An. Ci. Paraguayos 7: 6 (colocou esta espécie e C. virgatus Vachal, 1904, na sinonímia de C. semicyaneus Spinola, 1851 [= Reedapis semicyanea, um Paracolletini]. - Friese, 1910. Zool. Jahrb. Syst. 29: 646 (ao descrever $C$. chalybaeus comparou-a com $C$. cyaneus reproduzindo a descrição).

Biologia. Schlindwein, 1995. Wildbienen und ihre Trachtpflanzen... Loasaceen: 87-88 (em flores de Schinus molle L. (Anacardiaceae), Berberis laurina Billb. (Berberidaceae), Adesmia riograndensis S. T. S. Miotto (Fabaceae), Glechon thymoides Spreng. (Lamiaceae), Blumenbachia insignis Schrader (Loasaceae) e Monnina cuneata A. St. Hil. (Polygalaceae).

delicatus Metz. MÉxICO, Guadalajara (localidade-tipo).

Colletes delicata Metz, 1910. Pomona C. Jour. Ent. 2: 203. Cinco síntipos machos depositados na OSUC.

Taxonomia. Metz, 1910: 203 (comparou com C. macconnelli Metz, 1910). 
dilatatus Metz. MÉXICO, Guadalajara (localidade-tipo).

Colletes dilatata Metz, 1910. Pomona C. Jour. Ent. 2: 194. Síntipos: três machos e quinze fêmeas depositados na OSUC.

desantisi nom. n. ARGENTINA, Mendoza, S. Ignacio (localidade-tipo).

Colletes similis Joergensen, 1912. Zool. Jahrb. Syst. 32: 96; nom. praeoc. Holótipo fêmea no MLP.

Colletes desantisi nom. n. para Colletes similis Joergensen, 1912 (non Colletes similis Schenck, 1853 nec Robertson, 1904).

Taxonomia. Joergensen, 1912: 96 (comparou-a com C. glycyrrhizae, descrita na mesma publicação). - Durante \& Díaz, 1998. Rev. Mus. La Plata (N. S.) (33): 8 (examinaram o holótipo fêmea).

eupogonites Moure. ARgEnTINA, Tucumán, Tacanas (localidade-tipo).

Colletes eupogonites Moure, 1949. Rev. Ent., Rio de Janeiro, 20 (1/3): 439.

Lectótipo macho, aqui designado, depositado no DZUP; quatro paralectótipos machos no DZUP e cinco no IML.

Taxonomia. Moure, 1949: 439 (comparou com C. petropolitanus Dalla Torre, 1896).

extesicornis Vachal. BRASIL (localidade-tipo).

Colletes extensicornis Vachal, 1909. Rev. d' Ent., Caen, 28: 53. Lectótipo macho no MNHP, aqui designado; foi examinado por J.S. Moure.

Taxonomia. Vachal, 1909: 53 (comparou com C. brevinodis Vachal, 1909 e comentou que um ou outro poderia ser o macho de C. rutilans Vachal, 1909).

flaminii Moure. CHILE, Temuco, Constitución (localidade-tipo).

Colletes flaminii Moure, 1956. Dusenia 7 (4): 203. Holótipo fêmea no DZUP.

Taxonomia. Moure, 1956: 204 (comentou sua semelhança com C. weiskei Friese, 1912).

frontalis Metz. MÉxICO, Guadalajara (localidade-tipo).

Colletes frontalis Metz, 1910. Pomona C. Jour. Ent. 2: 207. Quatro síntipos fêmeas depositados na OSUC.

Taxonomia. Metz, 1910: 208 (poderia ser $C$. griseus Smith, 1879).

fulvipes Spinola. CHILE (localidade-tipo).

Colletes fulvipes Spinola, 1851, in Gay. Hist. Fis. Pol. Chile Zool. 6: 225.

Lectótipo macho no MNHP, aqui designado; foi examinado por J.S. Moure.

Colletes fulpis [sic]; Dalla Torre, 1896. Cat. Hym. 10: 41 (lapsus). Colletes flavipes [sic]; Ruiz, 1935. Rev. Chilena Hist. Nat. 39: 271.

Taxonomia. Spinola, 1851: 225 (comentou ser rara nas províncias centrais do Chile). 
furfuraceus Holmberg. ARgentinA, Buenos Aires, Tandil, Cerro Claraz (localidade-tipo); Mendoza, Chacras de Coria. ParaguaI.

Colletes furfuracea Holmberg, 1886. Act. Ac. Ci. Cordoba 5 (3): 183. Holótipo fêmea provavelmente no MBR.

Colletes furfuracens [sic]; Schrottky, 1903. An. Soc. Ci. Argentina 55: 180. Colletes furfurasceus [sic]; Schlindwein, 1995. Wildbienen und ihre Trachtpflanzen... Loasaceen: 88.

Taxonomia. Strand, 1909. Deutsch. ent. Ztschr. p. 230 (ocorrência no Paraguai). - Friese, 1910. Zool. Jahrb. Syst. 29: 650 - 651 (notas e comparação com C. steinbachi Friese, 1910).

Biologia. Jensen-Haarup, 1908. Flora og Fauna 10: 100 (observou-a em Baccharis). - Joergensen, 1912. Zool. Jahrb. Syst. 32: 96 (numerosos machos e fêmeas visitando Tamarix africana, Larrea divaricata Cav., Sphaeralcea bonariensis Griseb., Gourliaea [sic] decorticans [= Geoffroea decorticans (Hook. \& Arn.) Burk.], Prosopis alpataco Phil., Lycium chilense Bert.; fêmeas em Senecio mendocinus Phil. e Parthenium hysterioides). - Joergensen, 1912. An. Mus., Nac. Buenos Aires 22: 300 (comentou ser muito abundante de outubro a maio em Cyclolepis genistoides Gill. ex D. Don., Senecio mendocinus, Parthenium hysterioides; Hoffmannseggia falcata [sic] [Hoffmanseggia falcaria Cav.], Prosopis alpataco, P. campestris, Acacia furcata Desv., Gourliaea [sic] decorticans [= Geoffroea decorticans], Tamarix africana, Larrea divaricata, Sphaeralcea bonariensis Griseb., Lycium chilense Bert., Solanum elaeagnifolium Cav.-Schlindwein, 1995. Wildbienen und ihre Trachtpflanzen... Loasaceen: 88 (coletou em flores de Blumenbachia insignis Schrader). Morfologia. Graf, 1968. Bol. Univ. Fed. Paraná, Zoologia 3 (3): 73, 76 (nota sobre o canal salivar).

gilvus Vachal. CHILË, Arica (localidade-tipo).

Colletes gilvus Vachal, 1909. Rev. d' Ent., Caen, 28: 56. Síntipos fêmea e macho no MNHP.

glycyrrhizae Joergensen. ArgentinA, Mendoza, S. Ignacio (localidade-tipo).

Colletes glycyrrhizae Joergensen, 1912. Zool. Jahrb. Syst. 32: 95. Lectóti-

po fêmea, aqui designado, e paralectótipo macho no MLP.

Biologia. Joergensen, 1912: 95 (sobre Glycyrrhiza astragalina Gill.).

Taxonomia. Durante \& Díaz, 1998. Rev. Mus. La Plata (N. S.) (33): 8 (examinaram os síntipos, a fêmea rotulada com etiqueta de lectótipo por Moure \& Ogloblin).

griseus Smith. MÉxICO, Oaxaca (localidade-tipo). nom. reval.

Colletes griseus Smith, 1879. Descr. N. Sp. Hym. Br. Mus. p 2. Lectótipo fêmea no BMNH, aqui designado; foi examinado por J.S. Moure.

Colletes grisellus Michener, 1989. Univ. Kansas Sci. Bull. 53 (11): 703;

nom. n. para Colletes griseus Smith 1879 non Monia grisea Westwood, 1875 (= Monidia grisea). 
guadalajarensis Metz. MÉXICO, Guadalaraja (localidade-tipo).

Colletes guadalajarensis Metz, 1910. Pomona C. Jour. Ent. 2: 198. Dois síntipos machos depositados na OSUC.

Taxonomia. Metz, 1910: 200 (comparou com C. prosopidis Cockerell, 1897, espécie neártica).

intricatus Smith. MÉXICO (localidade-tipo), Orizaba.

Colletes intricatus Smith, 1879. Descr. N. Sp. Hym. Br. Mus. p. 2. Holótipo fêmea no BMNH, examinado por J.S. Moure.

Taxonomia. Cockerell, 1899. Cat. Abejas de México p. 4 (ocorrência).

isthmicus Swenk. PANAMÁ, San Lorenzo (localidade-tipo).

Colletes isthmicus Swenk, 1930. Psyche 37: 219. Holótipo fêmea e alótipo no BMHN, conforme citação do autor.

Taxonomia. Swenk, 1930: 222 (considerou-a próxima de C. mexicanus Cresson, 1868).

joergenseni Friese. ARGENTINA, Mendoza (localidade-tipo), Tucumán.

Colletes jörgenseni Friese, 1910. Zool. Jahrb. Syst. 29: 650. Lectótipo macho no MNHU, coletado em Mendoza em 2. XI. 1906, aqui designado; foi examinado por J.S. Moure.

Taxonomia. Friese, 1910: 650 (comparou com C. catulus Vachal, 1904).

kerri Moure. BRASIL, Paraná, Araucária (localidade-tipo) $820 \mathrm{~m}$.

Colletes kerri Moure, 1956. Dusenia 7 (4): 197. Holótipo fêmea, alótipo macho e dois parátipos no DZUP.

Biologia. Moure, 1956: 199 (comentou que emergiram Isepeolus viperinus (Holmberg, 1886), de alguns casulos de C. kerri). - Michener \& Lange, 1957. Jour. Kansas Ent. Soc. 30 (2): 79-80, fig. 10 (descreveram o ninho, com dois ou três ramos contendo séries de células como em Colletes michenerianus Moure, 1956, citaram Isepeolus viperinus). - Michener et al., 1958: 10 (ninhos em barrancos terrosos).

langeanus Moure. BRASIL, Paraná, Curitiba (localidade-tipo), 900m.

Colletes langeana Moure, 1956. Dusenia 7 (4): 199. Holótipo fêmea e um parátipo no DZUP.

Taxonomia. Moure, 1956: 200 (comparou com Melipona quadrifasciata quadrifasciata Lepeletier, 1836, pelo colorido).

Biologia. Moure, 1956: 200 (em flores de araçá [Psidium sp.]).

laticeps Friese. CHILE, Concepción (localidade-tipo).

Colletes laticeps Friese, 1910. Zool. Jahrb. Syst. 29: 651. Lectótipo fêmea no MNHU, coletado em II. 1904 por P. Herbst, aqui designado; foi examinado por J.S. Moure.

Taxonomia. Friese, 1910: 651 (comparou com C. cognatus Spinola, 1851). 
Biologia. Friese, 1910: 651 (em flores de Baccharis, em janeiro e fevereiro). - Jaffuel \& Pirión, 1926. Rev. Chilena Hist. Nat. 30: 364 (observaram espécimens voando sobre Senecio rutaceus Phil.; e parasitados por Epeolus luctuosus [= Isepeolus luctuosus]). - Claude-Joseph, 1926. Ann. Sci. Nat., Zool., Paris, (10) 9: 134-136, fig. 15 (ninhos com galerias dicotômicas entrelaçadas, e no fundo de cada ramo uma célula membranosa, raramente duas; encontrou Isepeolus Cockerell, 1907, e Anthrax Scopoli, 1763 [Diptera, Bombyliidae] parasitando os ninhos). - Janvier, 1933. Ann. Sci. Nat., Zool, Paris (10) 16: 325 (um ninho parasitado por Isepeolus luctuosus, e observou Isepeolus triseriatus [= Melectoides triseriatus] voando sobre as colônias e se reproduzindo em seus ninhos). - Ruiz, 1944. Rev. Chilena Hist. Nat. 48: 201-202 (descreveu uma colônia enorme num espaço de 50 $\mathrm{m}^{2}$, no Chile, nas margens do rio Cautin, com os orifícios de entrada muito próximos uns dos outros, e muitos Epeolus luctuosus [=Isepeolus luctuosus] voando juntamente com as fêmeas de Colletes; viu também numerosos machos nas folhas de Aristotelia maqui [sic] [Aristotelia macqui L'Herit.], tanto na face dorsal como na ventral das folhas).

Morfologia. McGinley, 1981. Univ. Calif. Publ., Entomology 91: 90 (redescreveu a larva e comparou com as de $C$. araucariae e $C$. thoracicus Smith, 1853, esta última neártica).

lineatus Metz. MÉXICO, Guadalajara) (localidade-tipo).

Colletes lineata Metz, 1910. Pomona C. Jour. Ent. 2: 196. Holótipo macho depositado na OSUC.

longiceps Friese. ARGENTINA, Neuquén (localidade-tipo).

Colletes longipes Friese, 1910. Zool. Jahrb. Syst. 29: 649. Lectótipo macho no MNHU, coletado por Lendl em 1907, aqui designado; foi examinado por J.S. Moure.

Taxonomia. Friese, 1910: 649 (comparou com C. nasutus Smith, 1853, uma espécie paleártica).

lucens Vachal. CHILE, Concepción (localidade-tipo).

Colletes lucens Vachal, 1909. Rev. d' Ent., Caen, 28: 57. Síntipos fêmea e macho provavelmente no MNHP.

lycii Joergensen. ARgentinA, Mendoza (localidade-tipo), Pedregal, Chacras de Coria, San Ignacio, Potrerillos.

Colletes lycii Joergensen, 1912. Zool. Jahrb. Syst. 32: 96. Lectótipo macho aqui designado, e três paralectótipos, dois machos e uma fêmea no MLP.

Taxonomia. Durante \& Díaz, 1998. Rev. Mus. La Plata (N. S.) (33): 8 (examinaram os síntipos de Mendoza sendo um macho coletado em 11. XI. 1906 rotulado com etiqueta de lectótipo por Moure \& Ogloblin).

Biologia. Joergensen, 1912: 97 (sobre flores de Lycium chilense Bert., Larrea divaricata Cav., Prosopis alpataco Phil. e Hoffmannseggia falcata 
[sic] [Hoffmannseggia falcaria Cav.]). - Joergensen, 1912. An. Mus. Nac. Buenos Aires 22: 300 (repetiu a relação das plantas e acrescentou $P$. campestris).

macconnelli Metz. MÉXICO (localidade-tipo), Guadalajara.

Colletes macconnelli Metz, 1910. Pomona C. Jour. Ent. 2: 201. Três síntipos machos depositados na OSUC.

Taxonomia. Cockerell, 1924. Proc. Calif. Acad. Sci. 12 (27): 533, 534 (nota e ocorrência).

mastochila Moure. CHILE, Arica, Putre (localidade-tipo), 3650m. Peru, Tacna, Rio Sama.

Colletes mastochila Moure, 1956. Dusenia 7 (4): 206. Holótipo macho, alótipo fêmea e oito parátipos no DZUP.

meridionalis Schrottky. Brasil, Rio Grande do Sul (localidade-tipo); São Paulo, São Paulo (Vila Ema), Rio Claro; ARgEnTINA, Misiones. PARAGUAI.

Colletes rufipes meridionalis Schrottky, 1902. Rev. Mus. Paulista 5: 345. Dois síntipos fêmeas no MZSP.

Colletes meridionalis; Moure, 1945. Rev. Ent., Rio de Janeiro, 16: 394.

Taxonomia. - Schrottky, 1913. An. Soc. Ci. Arg. 75: 236 (distribuição geográfica: Misiones, Argentina; Paraguai e Brasil). - Moure, 1945: 394-395 (ocorrência, considerou C. meridionalis como espécie distinta de C. rufipes e relacionou caracteres comparativos entre as duas espécies).

Biologia. - Moure, 1945: 394 (em flores de girassol [Helianthus annuus L.]). Morfologia. Cruz Landin, 1968. Arq. Zool., São Paulo, 17 (3): 119, estampas 4, 23, 24 (Histologia e ultraestrutura das glândulas salivares). - Cruz Landin, 1973. Studia Ent. 16 (1/4): 209 a 213, tab. I (glândulas salivares).

mexicanus Cresson. MÉXICO, [Veracruz], Orizaba (localidade-tipo).

Colletes mexicana Cresson, 1868. Proc. Boston Soc. Nat. Hist. 12: 170. Holótipo fêmea na ANSP, examinado por J.S. Moure.

Taxonomia. Cockerell, 1899. Cat. Abejas de México p. 4 (comentou a semelhança desta espécie com C. submarginata Cresson, 1865, de Cuba). Cresson, 1916. Mem, Amer. Ent. Soc. 1: 108 (tipo em bom estado de conservação).

michenerianus Moure. BRASIL, Paraná, Guarapuava (localidade-tipo), 1200m; Curitiba, $900 \mathrm{~m}$,

Colletes micheneriana Moure, 1956. Dusenia 7 (4): 201. Holótipo fêmea, alótipo macho e dois parátipos no DZUP.

Colletes mochinerianus [sic]; Michener et al., 1958. Dusenia 8 (1): 4.

Taxonomia. Moure, 1956: 203 (comentou semelhança desta espécie com $C$. cyanea Holmberg, 1903 e C. cyanescens (Haliday, 1937)).

Biologia. Michener \& Lange, 1957. Jour. Kansas Ent. Soc. 30 (2): 77-79, figs 
6 a 9 (descreveram ninhos e comentaram que era a única espécie de Colletes conhecida a construir casulo de fibras, com o espaço entre as fibras preenchido com material amorfo). - Michener et al., 1958: 10 (observaram ninhos em barrancos terrosos). - Schlindwein, 1995. Wildbienen und ihre Trachtpflanzen... Loasaceen: 20, 88 (em flores de Berberis laurina Billb. (Berberidaceae). Morfologia. Graf, 1968. Bol. Univ. Fed. Paraná, Zoologia, 3 (3): 73, 76 (nota sobre o canal salivar). - McGuinley, 1981. Univ. Calif. Publ., Entomology 91: 90 (comparou a larva de C. michenerianus com a de $C$. thoracicus Smith, 1853, espécie neártica, ressaltando as diferenças).

mimincus Cockerell. Peru, [Lima] Matucana (localidade-tipo); [Huanuco] Tingo [Maria].

Colletes mininca Cockerell, 1914. Jour. N.Y. Ent. Soc. 22: 328. Lectótipo macho no AMNH, coletado em 1913, aqui designado; um paralectótipo macho no MCZ; examinados por J.S. Moure.

Taxonomia. Cockerell, 1926. Ann. Mag. Nat. Hist. (9) 17: 301 (ocorrência). Biologia. Cockerell, 1926: 301 (visitando flores de Malvaceae).

moctezumensis Metz. MÉXICO, Moctezuma (localidade-tipo).

Colletes moctezumensis Metz, 1910. Pomona C. Jour. Ent. 2: 200. Holótipo macho depositado na OSUC.

montefragus Raw. JAMAICA, St. Andrew, Strawberry Hill (localidade-tipo).

Colletes montefragus Raw, 1984. Revta. Bras. Ent. 28 (4): 492. Holótipo macho na coleção do autor, parátipos no BMNH, DZUP, USNM, SEMK; University Museum, Oxford University, Oxford e na coleção do autor.

Taxonomia. Raw, 1984: 494 (comparou com Colletes rugicollis nigrior Michener, 1954).

Biologia. Raw, 1984. Revta. Bras. Ent. 28 (4): 497-500, fig. 1(descreveu ninhos encontrados em barrancos aos lados de uma rodovia; observou adultos ativos de maio a julho e fêmeas de Epeolus rufoclypeus Fox, 1891 entrando nos ninhos).

motaguensis Cockerell. GUATEMALA, Quirigua (localidade-tipo).

Colletes motaguensis Cockerell, 1912. Ann. Mag. Nat. Hist. (8) 9: 564.

Lectótipo fêmea no AMNH, aqui designado; examinado por J.S. Moure.

Taxonomia. Cockerell, 1912: 565 (relacionou a espécie com C. nautlanus Cockerell, 1899 e C. senilis Smith, 1879 [= C. petropolitanus Dalla Torre, 1896]).

murinus Friese. PERU, Callanga (localidade-tipo).

Colletes murinus Friese, 1900. Ent. Nachr. 26: 184. Lectótipo fêmea no MNHU, coletado em 1898, aqui designado; foi examinado por J.S. Moure. 
musculus Friese. Argentina, Neuquén (localidade-tipo); Buenos Aires. Chile, Concepción.

Colletes musculus Friese, 1910. Zool. Jahrb. Syst. 29: 647. Lectótipo fêmea no MNHU, coletado por Lendl em 1907 em Neuquén, aqui designado. No mesmo museu J.S. Moure examinou três exemplares com etiqueta de tipo, coletados em Buenos Aires, em 15. XII. 1905, esta localidade não foi relacionada por Friese.

Taxonomia. Friese, 1910: 647 (comparou com C. murinus).

Biologia. Claude-Joseph, 1926. Ann. Sci. Nat., Zool., Paris, (10) 9: 133-134, fig. 14 (descreveu o ninho, com galeria em arco mais ou menos circular e ramificada). - Janvier, 1933. Ann. Sci. Nat., Zool, Paris, (10) 16: 326 (observou Epeoloides septemnotatus [= Isepeolus septemnotatus (Spinola, 1851)] e Isepeolus triseriatus [= Melectoides triseriatus (Friese, 1908)] em ninhos desta espécie). - Ruiz, 1944. Rev. Chilena Hist. Nat. 48: 215-216 (observou galerias que tinham uma entrada comum e algumas células com o parasita Epeolus triseriatus [= Melectoides triseriatus]).

nautlanus Cockerell. MÉXICO, Veracruz, Rio Nautla (localidade-tipo), San Rafael. Colletes nautlanus Cockerell, 1899. Entomologist: 154. Síntipo fêmea no USNM, examinado por J.S. Moure.

Taxonomia. Cockerell, 1899: 155 (menos preta que C. aethiops Cresson, 1868, C. mexicanus Cresson, 1868 e C. nudus Robertson, 1898 [espécie neártica]).

neuqueenensis Friese. ARgENTINA, Neuquén (localidade-tipo), Mendoza.

Colletes neuqueenensis Friese, 1910. Zool. Jahrb. Syst. 29: 649. Lectótipo fêmea no MNHU, coletado em 1907 por Lendl, aqui designado; foi examinado por J.S. Moure.

Taxonomia. Friese, 1910: 649 (comparou com C. araucariae Friese, 1910).

niger Swenk. Costa RICA, Volcano Irazu (localidade-tipo).

Colletes niger Swenk, 1904. Can. Ent. 36: 76. Síntipos machos e fêmeas na UNL.

Biologia. Swenk, 1904: 77 (extremamente abundantes, de 8.500 a 9.500 pés, onde o solo é completamente perfurado pelos orifícios de entrada dos ninhos).

nigritulus Friese. Concepción, CHILE (localidade-tipo).

Colletes nigritulus Friese, 1910. Zool. Jahrb. Syst. 29: 645. Síntipo macho no MNHU, coletado por P. Herbst em X. 1903, examinado por J.S. Moure.

Taxonomia. Friese, 1910: 645 (comparou com C. bicolor Smith, 1879 e C. cyaniventris Spinola, 1851 [ = C. cyanescens (Haliday, 1837)).

Biologia. Jaffuel \& Pirión, 1926. Rev. Chilena Hist. Nat. 30: 364 (sobre Escallonia berteroana [sic] [ E. berteriana] [= Escallonia pulverulenta Pers.]). - Ruiz, 1944. Rev. Chilena Hist. Nat. 48: 217 (visitando flores de Quillaja saponaria Molina e Escallonia alba, nesta última coletando pólen). 
nitidicollis Friese. BolíviA, Jungas (localidade-tipo). PERU, Vilcanota.

Colletes nitidicollis Friese, 1900. Ent. Nachr. 26: 182. Síntipos fêmeas provavelmente no MNHU.

ornatus Schrottky. BRASIL, São Paulo, Jundiaí (localidade-tipo); Maranhão, Codó; Minas Gerais, Barbacena; Pará, Belém. Argentina, Misiones. Paraguai. COSTA RicA.

Colletes ornatus Schrottky, 1902. Rev. Mus. Paulista 5: 345. Holótipo fêmea no MZSP, M. Beron leg. 28. I. 1900.

Taxonomia. Schrottky, 1907. An. Ci. Paraguayos 7: 8 (ocorrência do sul do Paraguai até o Pará, Brasil). - Ducke, 1907. Rev. d'Ent., Caen, 26: 75 (ocorrência). - Schrottky, 1913. An. Soc. Ci. Argentina 75: 236 (ocorrência no Paraguai, Argentina e Brasil). - Friese, 1916. Stett, Ent, Ztg, 77: 290 (citou da Costa Rica).

panamensis Michener. PANAmÁ, Panamá (localidade-tipo), Las Sabanas; Coclé, El Valle de Antón, 2500 pés.

Colletes panamensis Michener, 1954. Bull. Amer. Mus. Nat. Hist. 104 (1):

20. Holótipo macho no AMNH. Alótipo, quatro parátipos fêmeas e um parátipo macho no AMNH e SEMK.

Taxonomia. Michener, 1954: 10, 20 (considerou-a próxima de C. submarginata Cresson, 1865).

Biologia. Michener, 1954: 21 (holótipo coletado em Cornuta [sic] grandifloria [sic] [Cornutia grandiflora Steud.]; alótipo e parátipos coletados em Physalis L.).

patagonicus Schrottky. ARGENTINA, Patagonia, Rio Caleufú (localidade-tipo).

Colletes patagonicus Schrottky, 1907. An. Ci. Paraguayos 7: 7. Síntipo fêmea, não examinado. Desconhecido o depositário.

Taxonomia. Cockerell, 1918: 138 (comparou C. rufosignatus com C. patagonicus).

perplexus Smith. MÉXICO (localidade-tipo), Orizaba. HonduRAS, Zamorano.

Colletes perplexus Smith, 1879. Descr. N. Sp. Hym. Br. Mus. p. 1. Lectótipo fêmea, no BMNH, aqui designado; foi examinado por J.S. Moure. Taxonomia. Cockerell, 1949. Proc. U.S. Nat. Mus. 98: 431 (ocorrência).

peruvicus Cockerell. PERU, Piura (localidade-tipo).

Colletes (lycii subsp) [sic] peruvicus Cockerell, 1913. Ann. Mag. Nat. Hist.

(8) 11: 185. Lectótipo fêmea no AMNH, aqui designado; foi examinado por J.S. Moure.

Colletes peruvicus Cockerell, 1913: 185.

Taxonomia. Cockerell, 1913: 185 (comparou com C. lycii Joergensen, 1912, de Mendoza e C. sulcatus Vachal, 1909, do Chile, e propôs uma chave para as espécies de Colletes do Peru: C. striginasis Vachal, 1909, C. inflatus Vachal, 1909 [= C. petropolitanus Dalla Torre, 1896] e C. peruvicus). 
petropolitanus Dalla Torre. BRASIL, Rio de Janeiro, Petrópolis (localidade-tipo); Pará; Maranhão, Codó; Ceará, Serra de Baturité; Mato Grosso, Salobra; Minas Gerais; Goiás; São Paulo; Paraná; Rio Grande do Sul. Peru (localidade-tipo de $C$. inflatus), Callanga. Bolívia, Mapiri, Colopampa. ArgentinA, Tucumán (localidade-tipo de C. catulus), Tacanas; Misiones; Mendoza, San Ignacio; Buenos Aires. PARAguaI, San Bernardino (localidade-tipo de $C$. speculiventris), San Estanislao.

Colletes senilis Smith, 1879. Descr. N. Sp. Hym. Br. Mus. p. 3; nom. praeoc. Lectótipo fêmea, aqui designado, no BMNH, 17. A. 533, coletado em Petrópolis em fevereiro de 1857; foi examinado por J.S. Moure.

Colletes petropolitanus Dalla Torre, 1896. Cat. Hym. 10: 43; nom. n. para Colletes senilis Smith (non Eversmann, 1854), - Moure, 1949. Rev. Ent. 20 (1/3): 437; syn.: C. catulus, $C$. inflatus, C. speculiventris.

Colletes catulus Vachal, 1904. Rev. d' Ent., Caen, 23: 26. Holótipo macho no MNHP, examinado por J.S. Moure.

Colletes inflatus Vachal, 1909. Rev. d' Ent., Caen, 28: 49. Síntipos do Peru e Bolívia provavelmente no MNHP.

Colletes apudeatulus [sic]; Joergensen, 1912. Zool. Jahrb. Syst. 32: 97.

Colletes apudcatulus [sic]; Joergensen, 1912. An. Mus. Nac. Buenos Aires 22: 301.

Colletes speculiventris Cockerell, 1917. Ann. Mag. Nat. Hist. (8) 20: 438.

Lectótipo macho no USNM, aqui designado, examinado por J.S. Moure.

Taxonomia. Smith, 1879 p. 3 (citou as duas localidades, Pará e Petrópolis). - Dalla Torre, 1896: 43 (citou Am. [América]: Parana [sic]). - Schrottky, 1902. Rev. Mus. Paulista 5: 346 (redescreveu a fêmea e o macho). Joergensen, 1912. Zool. Jahrb. Syst. 32: 97 (ocorrência). - Cockerell, 1917: 438 (ao descrever $C$. speculiventris comparou com $C$. argentinus Friese,1908). - Moure, 1949: 437-438 (sinonímia e ocorrência; comentou o erro de grafia de Joergensen, 1912).

Biologia. Ducke, 1910. Rev. Trim. Ceará 24: 44. (visitando flores de Borreria G. Mey.).

pinnatus Vachal. ARGENTINA, Mendoza (localidade-tipo),

Colletes pinnatus Vachal, 1909. Rev. d' Ent., Caen, 28: 51. Lectótipo macho no MNHP, aqui designado; foi examinado por J.S. Moure.

Taxonomia. Vachal, 1909: 51 (comparou macho e fêmea com C. enodis Vachal, 1909 [= Mourecotelles enodis (Vachal, 1909)]).

punctipennis Cresson. MÉXICO, Orizaba (localidade-tipo); Chichén Itzá, Yucatán. GuATEMALA.

Colletes punctipennis Cresson, 1868. Proc. Boston Soc. Nat. Hist. 12: 169.

Síntipo fêmea na ANSP, examinado por J.S. Moure.

Taxonomia. Cresson, 1916. Mem. Amer. Ent. Soc. 1: 109 (tipo em bom estado). - Cockerell, 1917. Ent. News 28: 363 (referiu-se ao macho de $C$. 
punctipennis coletado na Guatemala). - Schwarz, 1934. Amer. Mus. Novitates 72: 2 (ocorrência no México e notas descritivas).

quadrigenis Vachal. ARGENTINA, Tucumán, Tapia (localidade-tipo) 600m.

Colletes quadrigenis Vachal, 1909. Rev. d' Ent., Caen, 28: 51. Holótipo fêmea no MNHP, examinado por J.S. Moure.

Taxonomia. Moure, 1949. Rev. Ent., Rio de Janeiro, 20 (1/3): 439 (redescreveu e comparou com C. ornatus Schrottky, 1902).

recurvatus Metz. MÉXICO, Guadalajara (localidade-tipo).

Colletes recurvata Metz, 1910. Pomona C. Jour. Ent. 2: 192. Síntipos machos e fêmeas depositados na OSUC.

rhodaspis Cockerell. ARGENTINA, Chubut, Vale del Lago Blanco (localidade-tipo). CHILE, Tolhuaca, Mavihuan (localidade-tipo de C. campoi).

Colletes rhodaspis Cockerell, 1909. Ann. Mag. Nat. Hist. (8) 4: 397. Síntipo fêmea no BMNH, examinado por J.S. Moure.

Colletes campoi Herbst, 1920. Rev. Chilena Hist. Nat. 24: 8. Síntipo fêmea no MCZ, examinado por J.S. Moure. Syn. $\mathbf{n}$.

Taxonomia. Ruiz, 1944. Rev. Chilena Hist. Nat. 48: 205 (comparou com C. bicolor Smith, 1879).

Biologia. Ruiz, 1944: 205-206 (observou fêmeas fazendo galerias).

rohweri Cockerell. EQUADOR, Zaruma (localidade-tipo).

Colletes rohweri Cockerell, 1919. Proc. Ent. Soc. Wash. 20: 206. Holótipo macho no AMNH, coletado em 1915, examinado por J.S. Moure.

Taxonomia. Cockerell, 1919: 207 (comparou com C. intermixtus Swenk, 1905, uma espécie neártica).

rubicola Benoist. EQUADOR, Quito (localidade-tipo), Rumipamba, Linca, Vale de Lloa, El Angel (entre Ibarra e Tulcan),

Colletes rubicola Benoist, 1942. Ann. Soc. Ent. France 111: 76. Síntipos fêmeas e machos provavelmente no MNHP.

Biologia. Benoist, 1942: 78 (encontrou ninhos em troncos secos, com as células forradas internamente com uma película de celofane e dispostas em série linear; observou exemplares visitando diversas flores e em particular Dalea astragalina $\mathrm{H}$. B. K.).

rufipes Smith. BRASIL, Bahia (localidade-tipo); Ceará, Serra de Baturité, Quixadá; Mato Grosso, Salobra; São Paulo, Jundiaí, Campinas.

Colletes rufipes Smith 1879. Descr. N. Sp. Hym. Br. Mus. p. 3. Síntipo fêmea não examinado, provavelmente no BMNH.

Taxonomia. Schrottky, 1902: 344 (redescreveu a fêmea).

Biologia. Schrottky, 1907. An. Ci. Paraguayos 7 (1): 5 (observou exemplares em flores de Vernonia Schreb.). - Ducke, 1910. Rev. Trim. Ceará: 43 (visitando flores de Sapindus L., Serjania P. Miller e Borreria G. Mey.). 
rufosignatus Cockerell. ARgENTINA, Chubut (localidade-tipo), Patagonia.

Colletes rufosignatus Cockerell, 1918. Can. Ent. 50: 138. Síntipo macho no USNM, examinado por J.S. Moure.

Taxonomia. Cockerell, 1918: 138 (próxima de C. patagonicus Schrottky, 1907 e de C. rhodaspis Cockerell, 1909).

rugicollis Friese. BolíviA, Jungas (localidade-tipo), Santa Cruz. PerU. ARgENTINA, Tucumán; Buenos Aires. PARAgUAI, Asunción. BRASIL, Rio de Janeiro; São Paulo, Jundiaí (localidade-tipo de C. punctatissima), Rio Claro, São Paulo (Vila Ema); Paraná, Curitiba, São José dos Pinhais; Santa Catarina, Blumenau, Seara [Nova Teutonia]. Argentina, Mendoza (localidade-tipo de $C$. argentinus). PARAgUaI. COSTA RICA, San José.

Colletes rugicollis Friese, 1900. Ent. Nachr. 26: 183. Lectótipo fêmea no MNHU, aqui designado; foi examinado por J.S. Moure. - Moure, 1944. Rev. Ent. 15 (1/2): 2; syn. C. punctatissimus.

Colletes punctatissima Schrottky, 1902. Rev. Mus. Paulista 5: 347. Holótipo macho no MZSP (ex Museu Paulista), M. Beron leg. 28. I. 1900; examinado por J.S. Moure. Na descrição o autor colocou o símbolo de fêmea.

Colletes argentinus Friese, 1908. Flora og Fauna 10: 10. Lectótipo macho no MNHU, coletado em 10.II.1907, aqui designado; foi examinado por J.S. Moure. Syn. n.

Taxonomia. Schrottky, 1902: 347 (considerou Colletes punctatissima e $C$. rugicollis Friese, 1900 muito parecidas; redescreveu a fêmea de C. rugicollis, citou sua ocorrência na Bolívia e em Santa Catarina). - Friese, 1908: 11 (comparou C. argentinus com C. rugicollis). - Schrottky, 1913. An. Soc. Ci. Arg. 75: 237. (ocorrência na Argentina, Paraguai, Bolívia e Brasil). Friese, 1916. Stett. Ent. Ztg. 77: 290 (na Costa Rica e Peru). - Moure, 1949: 438 (ocorrência e redescrição). - Sakagami \& Laroca, 1971. Kontyû 39 (3): 219 (ocorrência em Curitiba e S. José dos Pinhais).

Biologia. Jensen-Haarup, 1908. Flora og Fauna 10: 100 (principalmente em Solanaceae). - Joergensen, 1912. Zool. Jahrb. Syst. 32: 99 (visitando Baccharis salicifolia Person). - Joergensen, 1912. An. Mus. Nac. Buenos Aires 22: 301 (bastante comum de novembro a abril em flores de Baccharis salicifolia, B. serrulata (Lam.) Person, Hoffmannseggia falcata [sic] [Hoffmannseggia falcaria Cav.]). - Friese, 1921. Stett. Ent. Ztg. 82: 75 (em Physalis). - Michener et. al., 1958. Dusenia 8 (1): 4 (ninhos em barrancos).

rugicollis nigrior Michener. PANAMÁ, Coclé, El Valle de Antón, 2500 pés (localidade-tipo); Chiriquí, Potrerillos.

Colletes rugicollis nigrior Michener, 1954. Bull. Amer. Mus. Nat. Hist.

104 (1): 17. Holótipo macho no AMNH, alótipo fêmea e três parátipos machos no AMNH e SEMK; dois parátipos, um macho e uma fêmea no North Carolina State College, Raleigh, N. C., Estados Unidos. 
rutilans Vachal. EQUAdOR (localidade-tipo). PERU. CHILE.

Colletes rutilans Vachal, 1909. Rev. d' Ent., Caen, 28: 52. Holótipo fêmea provavelmente no MNHP.

schrottkyi Joergensen. ARgentina, Mendoza, Chacras de Coria (localidade-tipo), San Ignacio.

Colletes schrottkyi Joergensen, 1912. Zool. Jahrb. Syst. 32: 97. Lectótipo macho, coletado por Joergensen, aqui designado e um paralectótipo fêmea, no MLP, examinados por J.S. Moure.

Taxonomia. Durante \& Díaz, 1998. Rev. Mus. La Plata (N. S.) (33): 8 (síntipos: macho rotulado com etiqueta de lectótipo por Moure \& Ogloblin e fêmea sem o tagma abdominal).

Biologia. Joergensen, 1912: 98 (observou grandes colônias nas paredes de barro de casas de campo ou em bambú). - Joergensen, 1912. An. Mus. Nac. Buenos Aires 22: 301 (abundante em outubro e dezembro sobre Patagonium gilliesii [sic] [= Adesmia giliesii Hook. \& Arn.]).

seminitidus Spinola. CHILE (localidade-tipo), Concepción, Nuble, Quillota. Colletes semi-nitida Spinola, 1851, in Gay. Hist. Fis. Pol. Chile, Zool. 6:

225. Síntipo fêmea no IZUT, examinado por J.S. Moure.

Taxonomia. Spinola: 226 (comparou com Colletes cyaniventris $[=C$. cyanescens]).

Biologia. Jaffuel \& Pirión, 1926. Rev. Chilena Hist. Nat. 30: 364 (exemplares parasitados por Epeoloides septemnotatus [= Isepeolus septemnotatus]). - Claude-Joseph, 1926. Ann. Sci. Nat., Zool., Paris, (10) 9: 130-131, fig. 12 (Coletou adultos sobre Colletia ferox Gill. \& Hook., Talguena[sic] costata [Talguenea costata Miers], Maytenus boaria Molina, Aristotelia maqui [sic] L'Herit., Porliera hygrometrica Ruiz \& Pavon; observou ninhos em todo o tipo de terreno, com uma entrada e quatro ou cinco galerias divergentes, cada uma com meia dúzia de células lineares).

spilopterus Cockerell. Panamá, Zona do Canal, Barro Colorado, Porto Bello (localidade-tipo), Coclé, El Valle de Antón; Chiriquí, Potrerillos. Costa RICA, San José (localidade-tipo de C. maculipennis), San Mateo.

Colletes spiloptera Cockerell, 1917. Ent. News 28: 363. Lectótipo macho no USNM, aqui designado; foi examinado por J.S. Moure. - Michener, 1989. Univ. Kansas Sci. Bull. 53 (11): 685; syn.: C. maculipennis.

Colletes (Ptilopoda) maculipennis Friese, 1921. Stett. Ent. Ztg. 82: 83.

Síntipos machos provavelmente no MNHU, coletados por Schmidt em 1. VII. 1920.

Taxonomia. Cockerell, 1917: 363 (comparou com C. punctipennis Cresson, 1868, de Orizaba, México) - Schwarz, 1934. Amer. Mus. Novit. 722: 2 (colocou C. maculipennis como sinônimo provável de C. spiloptera). Michener, 1954. Bull. Amer. Mus. Nat. Hist. 104: 21(examinou o tipo de C. spiloptera, e relatou novas ocorrências).

Biologia. Friese, 1925. Stett. Ent. Ztg. 86: 3 (em Physalis). 
steinbachi Friese. ARGENTINA, Salta (localidade-tipo).

Colletes steinbachi Friese, 1910. Zool. Jahrb. Syst. 29: 651. Lectótipo fêmea no MNHU, coletado em Salta, 2500m, em III. 1905, aqui designado; foi examinado por J.S. Moure.

Taxonomia. Friese, 1910: 651 (comparou com C. furfuraceus Holmberg, 1886).

striginasis Vachal. PERU, Urubamba, 3000m (localidade-tipo).

Colletes striginasis Vachal, 1909. Rev. d' Ent., Caen, 28: 48. Holótipo fêmea no MNHP, examinado por J.S. Moure.

subdilatatus Metz. MÉXICO (localidade-tipo).

Colletes subdilatata Metz, 1910. Pomona C. Jour. Ent. 2: 204. Três síntipos fêmeas depositados na OSUC.

Taxonomia. Metz, 1910: 204 (comparou com C. macconnelli Metz, 1910 e C. delicata Metz, 1910).

submarginatus Cresson. CUBA (localidade-tipo).

Colletes submarginta Cresson, 1865. Proc. Ent. Soc. Philadelphia 4: 167. Holótipo fêmea na ANSP, examinado por J.S. Moure.

Colletes subemarginatus [sic]; Ashmead, 1900. Tr. Ent. Soc. London, 2: 305.

sulcatus Vachal. CHILE. (localidade-tipo).

Colletes sulcatus Vachal, 1909. Rev. d' Ent., Caen, 28: 58. Lectótipo macho no MNHP, aqui designado; foi examinado por J.S. Moure.

tingoensis Cockerell. PERU, [Huanuco], Tingo [Maria] (localidade-tipo).

Colletes tingoensis Cockerell, 1926. Ann. Mag. Nat. Hist. (9) 17: 302.

Lectótipo macho no USNM, aqui designado; foi examinado por J.S. Moure.

Taxonomia. Cockerell, 1926: 302 (comparou com C. longiceps Friese, 1910).

tomentosus Friese. CHILE, Arica (localidade-tipo).

Colletes tomentosus Friese, 1910. Zool. Jahrb. Syst. 29: 648. Lectótipo fêmea no MNHU, aqui designado; foi examinado por J.S. Moure.

vachali Joergensen. ARgEnTINA, Mendoza (localidade-tipo), Chacras de Coria, San Ignacio

Colletes vachali Joergensen, 1912. Zool. Jahrb. Syst. 32: 93. Lectótipo macho, aqui designado, e um paralectótipo macho no MLP; examinados por J.S. Moure.

Taxonomia. Durante \& Díaz, 1998. Rev. Mus. La Plata (N. S.) (33): 8-9 (examinaram os síntipos, um dos machos rotulado como lectótipo por Moure \& Ogloblin).

Biologia. Joergensen, 1912: 94 (voando sobre Prosopis alpataco Phil. $e$ Prosopis campestris Gris.). - Joergensen, 1912. An. Mus. Nac. Buenos Aires 22: 300 (repetiu a citação das plantas). 
virgatus Vachal. ARgentinA, Carcarañá (localidade-tipo); Buenos Aires, La Plata; Neuquén.

Colletes virgatus Vachal 1904. Rev. d' Ent., Caen, 23: 26. Lectótipo macho no MNHP, aqui designado; foi examinado por J.S. Moure.

Taxonomia. Friese, 1910: 646 (comparou com C. chalybaeus Friese, 1910). volsellatus Metz. MÉXICO, Guadalajara (localidade-tipo).

Colletes volsellata Metz, 1910. Pomona C. Jour. Ent. 2: 197. Dois síntipos machos depositados na OSUC.

weiskei Friese. ARgENTINA, Neuquén (localidade-tipo), Cordillera.

Colletes weiskei Friese, 1912. Deutsch. ent. Ztschr. p. 364. Lectótipo fêmea no MNHU, coletado por E. Weiske em XI.1910, aqui designado; foi examinado por J.S. Moure.

Taxonomia. Friese, 1912: 364 (comparou com C. bicolor Smith, 1879).

\section{Hemicotelles Toro \& Cabezas, 1977, gen. reval.}

Hemicotelles Toro \& Cabezas, 1977. An. Mus Hist. Nat. Valparaíso 10: 46. Espécie-tipo: Lonchopria ruizii Herbst, 1923. Designação original.

Mourecotelles (Hemicotelles); Michener, 1989. Univ. Kansas Sci. Bull. 53(11): 683. - Michener, 2000. The Bees of the World: 159.

magallanes Toro \& Cabezas. ARgEntinA, Santa Cruz, Los Antiguos (localidadetipo). CHILE, Aisén, Chile Chico.

Hemicotelles magallanes Toro \& Cabezas, 1977. An. Mus Hist. Nat. Valparaíso 10: 48. Holótipo macho, alótipo fêmea na Coleção Toro. Um parátipo macho na CAS.

ruizii (Herbst). CHILE, Valparaíso (localidade-tipo), Coquimbo, Las Breas, Vicuña, Incahuasi.

Lonchopria ruizii Herbst, 1923. Rev. Chilena Hist. Nat. 27: 75. Síntipos

fêmeas na Coleção Herbst, provavelmente no MCZ.

Leioproctus (Lonchopria) ruizi [sic]; Michener, 1965. Bull. Amer. Mus. Nat. Hist. 130: 41.

Hemicotelles ruizii; Toro \& Cabezas, 1977. An. Mus Hist. Nat. Valparaíso 10: 47 .

Biologia. Herbst, 1923: 75, 76 (coletou fêmeas com pólen de Baccharis concava Person).

\section{Monidia Cockerell, 1905, gen. reval.}

Monia Westwood, 1875. Trans. Ent. Soc. London: 221; nom. praeoc. Espécie-tipo: Monia grisea Westwood, 1875, Monotípico.

Monidia Cockerell, 1905. Ent. News 16: 9; nom.n. para Monia Westwood non Gray, 1850. - Michener, 1989. Univ. Kansas Sci. Bull. 53 (11): 685 (= Colletes Latreille).

grisea (Westwood). MÉXICO (localidade-tipo).

Monia grisea Westwood, 1875. Trans. Ent. Soc. London: 222. Lectótipo

Revta bras. Zool. 19 (1): 1 - 30, 2002 
macho no Hope Museum, Oxford, aqui designado; foi examinado por J.S. Moure.

Monidia grisea; Cockerell, 1905. Ent. News 16: 9.

\section{Mourecotelles Toro \& Cabezas, 1977}

Mourecotelles Toro \& Cabezas, 1977. An. Mus Hist. Nat. Valparaíso 10: 50. - Michener, 1989. Univ. Kansas Sci. Bull. 53 (11): 683; sub-gen.: Hemicotelles e Xanthocotelles. Espécie-tipo: Mourecotelles mixta Toro \& Cabezas, 1977.

biciliatus (Cockerell). CHILE, Concepción (localidade-tipo). comb. $\mathbf{n}$.

Colletes ciliatus Friese, 1910. Zool. Jahrb. Syst. 29: 647; nom. praeoc.

Lectótipo macho no MNHU, coletado em 7. X. 1908, aqui designado; dois paralectótipos no AMNH, o macho coletado em 17. X. 1908 e a fêmea em 5. XI. 1908; foram examinados por J.S. Moure.

Colletes biciliatus Cockerell, 1918. Can. Ent. 50: 138; nom. n. para Colletes ciliatus Friese, 1910, non Colletes ciliatus Patton, 1879.

Colletes polynidus Stephen, 1953. Dusenia 4 (1): 40; nom. n. para Colletes ciliatus Friese, 1910, non Patton.

Taxonomia. Friese, 1910: 647 (comparou com C. semicyaneus [= Reedapis semicyanea (Spinola, 1851), um Paracolletini] e C. musculus Friese, 1910). Biologia. Jaffuel \& Pirión, 1926. Rev. Chilena Hist. Nat. 30: 364 (parasitada por Epeolus triseriatus [= Melectoides triseriatus]). - Claude-Joseph, 1926. Ann. Sci. Nat., Zool., Paris, (10) 9: 136-139, figs, 16, 17 (sobre flores de Berberis chilensis Gill., Adesmia arborea Bert. e Phacelia circinata Jacq.; observou que as fêmeas procuravam ninhos velhos de Odynerus humeralis [= Hypodynerus humeralis (Haliday, 1836)], onde muitos indivíduos partilhavam as diferentes células do ninho protetor, também em galerias abertas por xilófagos em ramos secos de Peumus Molina e Kagenectia [sic] [Kagenneckia Ruiz \& Pavon], que ficaram crivados de aberturas circulares). - Ruiz, 1944. Rev. Chilena Hist. Nat. 48: 209 (referiu-se ao uso de ninhos velhos de Odynerus Latreille, 1802 e de Hypodynerus chilensis (Spinola, 1851)).

boliviensis Toro \& Cabezas. BolíviA, Camargo (localidade-tipo). ARgEnTINA, Tres Cruces.

Mourecotelles boliviensis Toro \& Cabezas, 1977. An. Mus Hist. Nat. Valparaíso 10: 58. Holótipo e alótipo e um parátipo macho no SEMK. Dois parátipos, um macho e uma fêmea, na Coleção Toro.

chillan Toro \& Cabezas. CHILE, Nuble, Las Trancas (localidade-tipo), Las Cabras; Osorno, Puyehue.

Mourecotelles chillan Toro \& Cabezas, 1977. An. Mus Hist. Nat. Valparaíso 10: 53. Holótipo macho e alótipo fêmea na Coleção Toro. Parátipos na UCVC e CAS.

enodis (Vachal). ARGENTINA, Mendoza (localidade-tipo).

Colletes enodis Vachal, 1909. Rev. d' Ent., Caen, 28: 50. Lectótipo macho no MNHP, aqui designado; foi examinado por J.S. Moure. 
Mourecotelles enodis; Toro \& Cabezas, 1977. An. Mus Hist. Nat. Valparaíso 10: 61 .

Taxonomia. Vachal, 1909: 50 (descreveu macho e fêmea). - Toro \& Cabezas, 1977: 61 (examinaram um macho da Coleção Vachal, atualmente depositada no MNHP, com etiqueta de Holótipo).

mixtus Toro \& Cabezas. ChILE, Valparaíso, Colliguay (localidade-tipo); Coquimbo.

Mourecotelles mixta Toro \& Cabezas, 1977. An. Mus Hist. Nat. Valparaíso 10: 52. Holótipo macho e alótipo fêmea na Coleção Toro. Numerosos parátipos machos e fêmeas no UCVC, MHNS, SEMK, AMNH, DZUP e Universidade da Califórnia.

moldenkei Toro \& Cabezas. CHILE, Aisén, Chile Chico (localidade-tipo); Magallanes, Brazo Norte.

Mourecotelles moldenkei Toro \& Cabezas, 1977. An. Mus Hist. Nat. Valparaíso 10: 55. Holótipo macho e alótipo fêmea na Coleção Toro. Parátipos na UCVC e CAS.

puelche Toro \& Cabezas. CHILE, Nuble, Las Trancas (localidade-tipo).

Mourecotelles puelche Toro \& Cabezas, 1977. An. Mus Hist. Nat. Valparaíso 10: 58. Holótipo macho na Coleção Toro. Um parátipo macho no SEMK.

spinolae (Crawford \& Titus). BolíviA, La Paz (localidade-tipo).

Diphaglossa spinolae Crawford \& Titus, 1904. Can. Ent. 36: 50. Sintípo macho no USNM, coletado em 14. XI. 1898.

Mourecotelles spinolae; Toro \& Cabezas, 1977. An. Mus Hist. Nat. Valparaíso 10: 60 .

Taxonomia. Toro \& Cabezas, 1977: 60-61. (redescreveram o síntipo).

triciliatus Toro \& Cabezas. Argentina, Catamarca, Aconquija (localidade-tipo). Mourecotelles triciliatus Toro \& Cabezas, 1977. An. Mus Hist. Nat. Valparaíso 10: 56. Holótipo macho e alótipo fêmea na Coleção Toro.

\section{Rhynchocolletes Moure, 1943, gen. reval.}

Rhynchocolletes Moure, 1943. Rev. Ent., Rio de janeiro, 14: 447. Espécie-tipo: Rhynchocolletes albicinctus Moure, 1943.

Colletes; Michener, 1989. Univ. Kansas Sci. Bull. 53 (11): 685; syn. Rhynchocolletes. - Michener, 2000. The Bees of the World: 135.

Taxonomia. Moure, 1943: 447 (descreveu o gênero e comparou com Diphaglossa Spinola, 1851, [Diphaglossinae]).

albicinctus Moure. BRASIL, Rio de Janeiro, Itatiaia (localidade-tipo) 700m.

Rhynchocolletes albicinctus Moure, 1943. Rev. Ent., Rio de Janeiro, 14:

447. Holótipo macho e um parátipo macho no DZUP.

Colletes albicinctus; Michener, 1989. Univ. Kansas Sci. Bull. 53 (11): 685.

Biologia. Moure, 1943: 447 (em flores de Camboatá [Guarea sp]). 


\section{Xanthocotelles Toro \& Cabezas, 1978, gen. reval.}

Xanthocotelles Toro \& Cabezas, 1978. An. Mus. Hist. Nat. Valparaíso 11: 131. Espécie-tipo: Xanthocotelles adesmiae Toro \& Cabezas, 1978.

Mourecotelles (Xanthocotelles); Michener, 1989. Univ. Kansas Sci. Bull. 53 (11): 683. - Michener, 2000. The Bees of the World: 159.

adesmiae Toro \& Cabezas. Chile, Valparaíso, Quilpué (localidade-tipo), Colliguay; Santiago, Talagante; Aconcágua, Catapilco.

Xanthocotelles adesmiae Toro \& Cabezas, 1978. An. Mus. Hist. Nat.

Valparaíso 11: 133. Holótipo macho e alótipo fêmea na Coleção Toro.

Parátipos no SEMK, AMNH, UCVC e Coleção Toro.

Biologia. Toro \& Cabezas, 1978: 134 (em flores de Adesmia DC.).

aisen Toro \& Cabezas. CHILE, Aisén, Chile Chico (localidade-tipo).

Xanthocotelles aisen Toro \& Cabezas, 1978. An. Mus. Hist. Nat. Valparaíso 11: 144. Holótipo macho na Coleção Toro.

andinus (Ruiz). CHILE, Coquimbo, Baños del Toro (localidade-tipo), Río Laguna

Colletes andina Ruiz, 1938. Rev. Chilena Hist. Nat. 42: 149. Holótipo

fêmea, alótipo macho, quatro parátipos machos e seis fêmeas na Coleção do Museu de História Natural do Colégio San Pedro Nolasco, Santiago, Chile.

Xanthocotelles andina; Toro \& Cabezas, 1978. An. Mus. Hist. Nat. Valparaíso 11: 139.

Taxonomia. Toro \& Cabezas, 1978: 139, figs 66 a 70 (redescreveram macho e fêmea).

atacama Toro \& Cabezas. CHILE, Atacama, Carrizal Bajo (localidade-tipo).

Xanthocotelles atacama Toro \& Cabezas, 1978. An. Mus. Hist. Nat. Valparaíso 11: 138. Holótipo e alótipo na Coleção Toro, um parátipo no SEMK.

basitarsalis Toro \& Cabezas. ARgEnTINA, Catamarca, Buena Vista (localidadetipo).

Xanthocotelles basitarsalis Toro \& Cabezas, 1978. An. Mus. Hist. Nat.

Valparaíso 11: 142. Holótipo macho no AMNH, um parátipo na Coleção Toro.

fritzi Toro \& Cabezas. CHILE, Nuble, las Trancas (localidade-tipo).

Xanthocotelles fritzi Toro \& Cabezas, 1978. An. Mus. Hist. Nat. Valparaíso 11: 137. Holótipo macho na Coleção Toro.

incahuasi Toro \& Cabezas. CHILE, Coquimbo, Incahuasi (localidade-tipo), Tres Cruces; Río Turbio.

Xanthocotelles incahuasi Toro \& Cabezas, 1978. An. Mus. Hist. Nat. Valparaíso 11: 136. Holótipo macho e alótipo fêmea na Coleção Toro.

Parátipos no SEMK, AMNH, UCVC, DZUP. 
plantaris (Vachal). ARgENTINA, Mendoza (localidade-tipo).

Colletes plantaris Vachal, 1909. Rev. d' Ent., Caen, 28: 53. Lectótipo macho no MNHP, aqui designado; um paralectótipo macho no MNHP; foram examinados por J.S. Moure.

Colletes plataris [sic]; Joergensen, 1912. Zool. Jahrb. Syst. 32: 99.

Xanthocotelles plantaris; Toro \& Cabezas, 1978. An. Mus. Hist. Nat. Valparaíso 11: 142.

sicheli (Vachal). CHILE (localidade-tipo), Nuble, Las Trancas, Las Cabras; Talca, Laguna del Maule; Chillán; Coquimbo, Baños del Toro.

Colletes sicheli Vachal, 1909. Rev. d' Ent., Caen, 28: 58. Lectótipo fêmea no MNHP, aqui designado; paralectótipo macho no MNHP; foram examinados por J.S. Moure.

Xanthocotelles sicheli; Toro \& Cabezas, 1978. An. Mus. Hist. Nat. Valparaíso 11: 134.

Taxonomia. Toro \& Cabezas, 1978: 134 (redescreveram macho e fêmea).

subandinus Toro \& Cabezas. CHILE, Aconcágua, Guardia Vieja (localidade-tipo). Xanthocotelles subandina Toro \& Cabezas, 1978. An. Mus. Hist. Nat. Valparaíso 11: 141. Holótipo macho e um parátipo macho na Coleção Toro.

tarsalis Toro \& Cabezas. ARgENTINA, Tres Cruces (localidade-tipo).

Xanthocotelles tarsalis Toro \& Cabezas, 1978. An. Mus. Hist. Nat. Valparaíso 11: 143. Holótipo macho no SEMK, parátipos na Coleção Toro.

\begin{abstract}
AGRADECIMENTOS. Ao Prof. Olavo Araujo Guimarães do Departamento de Botânica da Universidade Federal do Paraná, pela colaboração na nomenclatura das plantas; ao Prof. Charles D. Michener pelas informações sobre a Coleção Metz e ao Prof. Albino Morimasa Sakakibara pelo apoio nos procedimentos nomenclaturais.
\end{abstract}

\title{
REFERÊNCIAS BIBLIOGRÁFICAS
}

Durante, S. P. \& N. B. Díaz. 1998. Los ejemplares tipo de Apoidea (Hymenoptera) depositados en la coleccion del Museo de La Plata. Rev. Mus. La Plata N.S. (33): 1-14.

Friese, H. 1910. Zur Bienenfauna des südlichen Argentinien (Hym.). Zool. Jahrb. Syst. 29: 641-660. Joergensen, P. 1912. Los Crisídidos y los Himenópteros Aculeatos de la Provincia de Mendoza. An.

Mus. Nac. Buenos Aires 22: 267-338.

Metz, C.W. 1910. Bees of the genus Colletes from México. Pomona C. Jour. Ent. 2: 191-208.

MiChENER, C.D. 1944. Comparative external morphology, phylogeny, and a classification of the bees

Bull. Amer. Mus. Nat. Hist. 82: 151-326.

- 1989. Classification of the American Colletinae (Hymenoptera, Apoidea). Univ. Kansas Sci. Bull. 53 (11): 623-703.

2000 The Bees of the World. London, John Hopkins Univ. Press, I-XIV+913p.

Ruiz, F. 1944. Apidologia Chilena, II. Rev. Chilena Hist. Nat. 48: 200-231.

SCHLIndweIn, C. 1995. Wildbienen und ihre Trachtpflanzen in einer südbrasilianischen Buschlandschaft: Fallstudie Guaritas, Bestäubung bei Kakteen und Loasaceen. Stuttgart, Verlag Ulrich E. Grauer, 148p. 
Toro, H. \& V. Cabezas. 1977. Nuevos generos y especies de Colletini sudamericanos. Primera parte. An. Mus. Hist. Nat. Valparaíso 10: 45-64.

1978. Nuevos generos y especies de Colletini sudamericanos. Segunda parte. An. Mus. Hist. Nat. Valparaíso 11: 131-148.

VACHAL, J. 1909. Espèces nouvelles ou litigieuses d'Apidae du Haut Bassin du Parana et des Régions contigües et délimitation d'une nouvelle sous-familie Diphaglossinae (Hym.). Rev. d'Ent., Caen, 28: 5-64.

Recebido em 25.V.2001; aceito em 19.II.2002.

\section{Índice dos taxa de Colletini neotropicais}

adesmiae Toro \& Cabezas, Xanthocotelles......25

aethiops Cresson, Colletes .3

aisen Toro \& Cabezas, Xanthocotelles .............25

albicinctus Moure, Rhynchocolletes.................24

alocochila Moure, Colletes ................................... 3

andina $($ Joergensen $)=$ bicolor .............................. 5

andinus (Ruiz), Xanthocotelles ..........................25

antiguensis Cockerell, Colletes ............................ 3

apudcatulus $[\mathbf{s i c}]=$ petropolitanus ....................17

apudeatulus $[\mathbf{s i c}]=$ petropolitanus .....................17

araucariae Friese, Colletes ................................. 3

argentinus Friese $=$ rugicollis ...........................19

atacama Toro \& Cabezas, Xanthocotelles .......25

atacamanus Moure, Colletes................................. 4

atacamensis Janvier, Colletes ............................... 4

atacamensis Moure = atacamanus ..................... 4

atripes Smith, Colletes .......................................... 4

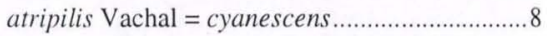

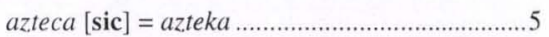

azteka Cresson, Colletes........................................ 4

azureus Friese, Colletes.......................................5

basitarsalis Toro \& Cabezas, Xanthocotelles ..25

biciliatus (Cockerell), Mourecotelles ................23

bicolor Smith, Colletes .......................................... 5

boliviensis Toro \& Cabezas, Mourecotelles.....23

bombiformis Metz, Colletes ................................. 6

brethesi Joergensen, Colletes ............................... 6

brevinodis Vachal, Colletes.................................. 6

bruneri Swenk, Colletes........................................ 6

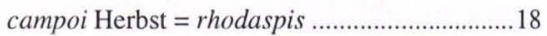

capitatus Metz, Colletes ....................................... 6

catulus Vachal = petropolitanus ...................... 17

chalybaeus Friese, Colletes .................................. 6

chillan Toro \& Cabezas, Mourecotelles ...........23

chubutensis Cockerell, Colletes ........................... 6

ciliatus Friese $=$ biciliatus .................................23

clarus Joergensen, Colletes ................................. 6

clematidis Joergensen, Colletes .......................... 7

cognatus Spinola, Colletes .................................. 7

costaricensis Friese, Colletes .............................. 7

Colletes Latreille ................................................... 2

cyaneus Holmberg, Colletes ................................ 8

cyanescens (Haliday), Colletes ............................. 7

cyaniventris Spinola = cyanescens ..................... 7

delicatus Metz, Colletes ....................................... 8

dilatatus Metz, Colletes ....................................... 9

desantisi Moure \& Urban, Colletes.................... 9

dimidiata Spinola $=$ atripes ................................ 4

enodis (Vachal), Mourecotelles ........................223

eupogonites Moure, Colletes............................... 9

extensicornis Vachal, Colletes ........................... 9

flaminii Moure, Colletes..................................... 9

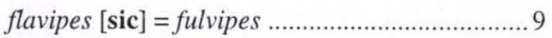

fritzi Toro \& Cabezas, Xanthocotelles ..............25

frontalis Metz, Colletes...................................... 9

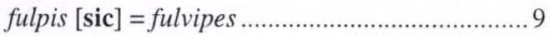

fulvipes Spinola, Colletes .................................. 9

furfuracens $[\mathbf{s i c}]=$ furfuraceus $\ldots \ldots \ldots \ldots \ldots \ldots \ldots \ldots . . . . . . .10$

furfuraceus Holmberg, Colletes ........................ 10

furfurasceus $[\mathbf{s i c}]=$ furfuraceus ........................ 10 
gilvus Vachal, Colletes ..................................... 10

glycyrrhyzae Joergensen, Colletes ....................10

griseus Smith, Colletes.................................... 10

grisea (Westwood), Monidia.............................22

grisellus Michener $=$ Colletes griseus ...............10

guadalajarensis Metz, Colletes.........................11

Hemicotelles Toro \& Cabezas............................22

incahuasi Toro \& Cabezas, Xanthocotelles .....25

inflatus $\mathrm{Vachal}=$ petropolitanus .......................17

intricatus Smith, Colletes ...................................11

isthmicus Swenk, Colletes ................................ 11

joergenseni Friese, Colletes ...............................11

kerri Moure, Colletes .........................................11

langeanus Moure, Colletes................................11

laticeps Friese, Colletes .....................................11

lineatus Metz, Colletes ..................................... 12

longiceps Friese, Colletes.................................. 12

lucens Vachal, Colletes ..................................... 12

lycii Joergensen, Colletes ................................... 12

macconnelli Metz, Colletes ................................13

maculipennis Friese = spilopterus .....................20

magallanes Toro \& Cabezas, Hemicotelles .....22

mastochila Moure, Colletes................................13

meridionalis Schrottky, Colletes ......................13

mexicanus Cresson, Colletes ............................ 13

michenerianus Moure, Colletes........................13

mimincus Cockerell, Colletes............................14

mixtus Toro \& Cabezas, Mourecotelles ............24

moctezumensis Metz, Colletes ......................... 14

moldenkei Toro \& Cabezas, Mourecotelles .....24

Monia Westwood = Monidia ............................22

Monidia Cockerell ..............................................22

montefragus Raw, Colletes .............................. 14

motaguensis Cockerell, Colletes ....................... 14

Mourecotelles Toro \& Cabezas .........................23

murinus Friese, Colletes .................................. 14

musculus Friese, Colletes................................. 15

nautlanus Cockerell, Colletes.............................15

neuqueenensis Friese, Colletes..........................15

niger Swenk, Colletes ..................................... 15

nigrior Michener, Colletes rugicollis ................ 19

nigritulus Friese, Colletes .................................. 15

nitidicollis Friese, Colletes ...............................16 ornatus Schrottky, Colletes............................... 16

panamensis Michener, Colletes ....................... 16

patagonicus Schrottky, Colletes ....................... 16

perplexus Smith, Colletes .................................. 16

peruvicus Cockerell, Colletes ........................... 16

petropolitanus Dalla Torre, Colletes ................ 17

pinnatus Vachal, Colletes .................................. 17

plantaris (Vachal), Xanthocotelles ...................26

plataris $[\mathbf{s i c}]=$ plantaris ................................. 26

polinydus $($ Stephen $)=$ biciliatus ....................... 23

puelche Toro \& Cabezas, Mourecotelles ......... 24

punctatissima $=$ rugicollis ............................... 19

punctipennis Cresson, Colletes ......................... 17

quadrigenis Vachal, Colletes ............................ 18

recurvatus Metz, Colletes ................................. 18

Rhynchocolletes Moure...................................... 24

rhodaspis Cockerell, Colletes ........................... 18

rohweri Cockerell, Colletes .............................. 18

rubicola Benoist, Colletes ................................ 18

rufipes Smith, Colletes ..................................... 18

rufipes meridionalis Schrottky $=$ Colletes meri-

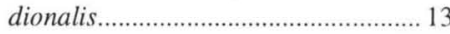

rufosignatus Cockerell, Colletes ...................... 19

rugicollis Friese, Colletes ................................ 19

ruizi $[$ sic]; Michener $=$ ruizii $\ldots \ldots \ldots \ldots \ldots \ldots \ldots \ldots . . . . . . . . . . . . .22$

ruizii (Herbst), Hemicotelles ............................. 22

rutilans Vachal, Colletes................................... 20

schrottkyi Joergensen, Colletes ......................... 20

seminitidus Spinola, Colletes ........................... 20

senilis Smith = petropolitanus ......................... 17

sicheli (Vachal), Xanthocotelles ...................... 26

similis Joergensen $[\mathrm{praeoc}]=$ desantisi .............. 9

speculiventris Cockerell = petropolitanus ....... 17

spilopterus Cockerell, Colletes .......................... 20

spinolae (Crawford \& Titus), Mourecotelles ... 24

steinbachi Friese, Colletes ................................. 21

striginasis Vachal, Colletes................................2 21

subandinus Toro \& Cabezas, Xanthocotelles .. 26

subdilatatus Metz, Colletes...............................21

submarginatus Cresson, Colletes .......................21

sulcatus Vachal, Colletes ...................................21

tarsalis Toro \& Cabezas, Xanthocotelles......... 26

tingoensis Cockerell, Colletes ............................ 21

tomentosus Friese, Colletes............................... 21 
triciliatus Toro \& Cabezas, Mourecotelles ......24 vachali Joergensen, Colletes ...............................21

virgatus Vachal, Colletes...................................22

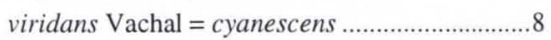

volsellatus Metz, Colletes..................................22

weiskei Friese, Colletes ....................................22

Xanthocotelles Toro \& Cabezas ........................25

\section{Índice das plantas}

Acacia furcata Desv. [Mimosaceae] .............5, 10

Acasia $[\mathbf{s i c}]$ furcata $=$ A. furcata ........................ 5

Adesmia DC. [Fabaceae] ...................................25

Adesmia arborea Bert. [Fabaceae]................4, 23

Adesmia giliesii Hook. \& Arn. [Fabaceae].......20

Adesmia riograndensis S.T.S. Miotto [Fabaceae]

...8

Alstroemeria L. [Amaryllidaceae].........................

Anacardiaceae .................................................... 8

Araçá [Psidium L.]

Aristotelia macqui L'Herit. [Tiliaceae] ......12, 20

Baccharis L. [Asteraceae] .... $8,10,12$

Baccharis concava Pers.[Asteraceae] ..............22

Baccharis pingraea DC. [Asteraceae] ................5

Baccharis pingrea $[\mathrm{sic}]$ angustissima $=$ B. pingraea

Baccharis rosmarinifolia Hook \& Arn. [Asteraceae]

Baccharis serrulata (Lam.) Pers. [Asteraceae]..... $.5,19$

Baccharis salicifolia Pers. [Asteraceae].......5, 19 Berberidaceae 8,14

Berberis chilensis Gill. [Berberidaceae] ..........23

Berberis laurina Billb.[Berberidaceae] ........8, 14 Blumenbachia insignis Schrader [Loasaceae].......

Borreria G. Mey. [Rubiaceae] ...................17, 18

Camboatá = Guarea sp....................................24

Clematis denticulata Vell. [Ranunculaceae] .......5

Clematis hilarii Spreng. $=$ C. denticulata $\ldots \ldots \ldots . . . .7$

Colletia sp. [Rhamnaceae]................................... 8

Colletia ferox Gill. \& Hook. [Rhamnaceae].....20

Colletia ulcina $[$ sic $]=$ C. ulicina .......................... 8

Colletia ulicina Gill. \& Hook. [Rhamnaceae]....8

Compostas [Asteraceae]
Cornutia grandiflora Steud. [Verbenaceae] .... 16

Cornuta $[\mathbf{s i c}]$ grandifloria $[\mathbf{s i c}]=$ Cornutia gran diflora ............................................ 16

Cyclolepis genistoides Gill ex D. Don. [Asteraceae] .................................................... 10

Dalea astragallina [Fabaceae] H. B. K. .......... 18

Deutzia crenata Sieb. \& Zucc. [Saxifragaceae]. 8

Erygium $[\mathbf{s i c}]$ paniculatum $=$ Eryngium panicula tum.

Eryngium paniculatum Cav. \& Dombay [Apiaceae].

Escallolia alba [Saxifragaceae] ........................ 15

Escallonia berteriana $=$ E. pulverulenta $\ldots \ldots \ldots . .15$

Escallonia berteroana $[\mathbf{s i c}]=$ E. pulverulenta .15

Escallonia illinita Presl.[Saxifragaceae] ............ 8

Escallonia pulverulenta Pers. [Saxifragaceae]. 15

Eugenia corralensis Phil. [Myrtaceae] ...............5

Fabaceae .......................................................... 8

Geoffroea decorticans [Fabaceae] ................... 10

Girassol [Helianthus annuus].......................... 13

Glechon thymoides Spreng. [Lamiaceae] .......... 8

Glycyrrhiza astragalina Gill. [Fabaceae].5, 7, 10

Gourliaea $[\mathrm{sic}]$ decorticans $=$ Geoffroea decorti cans. .................................................. 10

Guarea sp. [Meliaceae] ......................................24

Helianthus annuus L. [Asteraceae] ................... 13

Hoffmannseggia falcaria Cav. [Caesalpinaceae].. 10,19

Hoffmannseggia falcata $[\mathbf{s i c}]=H$. falcaria $\ldots \ldots \ldots . . .$. .. $10,12,19$

Kageneckia Ruiz \& Pavon [Rosaceae] .............23

Kagenectia $[\mathbf{s i c}]=$ Kageneckia .........................23

Labiatae $=$ Lamiaceae ...................................... 4

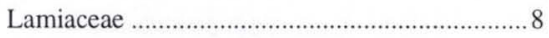

Larrea divaricata Cav. [Zygophyllaceae].. 10, 12

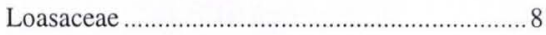

Lycium argentinum Hieron [Solanaceae]............5

Lycium chilense Bert.[Solanaceae] ........5, 10, 12

Lycium gracile $=$ Lycium chilense Bert...............5

Malvaceae ........................................................ 14

Maytenus boaria Molina [Celastraceae] ..........20

Medicago sativa L.[Fabaceae] ........................... 5

Monnina cuneata A. St. Hil. [Polygalaceae]....... 8 
Parthenium hysterioides $[\mathbf{s i c}]=P$. hysterophorus

Parthenium hysterophorus L. [Asteraceae] .....10 Patagonium giliesii $=$ Adesmia giliesii.........7, 20

Peumus Molina [Monimiaceae] .23

Phacelia circinata Jacq. [Hydrophyllaceae] ....23

Physalis L. [Solanaceae] 19,20

Physalis viscosa L. [Solanaceae] ........................ 5

Polygalaceae. .8

Porliera hygrometrica Ruiz \& Pavon [Zygophyllaceae]. 20

Prosopis alpataco Phil. [Mimosaceae] $.5,7,10,12,21$

Prosopis campestris Gris.

[Mimosaceae]. $5,7,10,13,21$

Psidium sp. [Myrtaceae]. .11

Quillaja saponaria Molina [Rosaceae]........8, 15

Sapindus L. [Sapindaceae] .18

Schinus molle L. [Anacardiaceae] …………….... 8

Senecio (Tourn.) L. [Asteraceae] ........................ 5

Senecio mendocinus Phil. [Asteraceae].............10

Senecio rutaceus Phil. [Asteraceae] .................. 12

Serjania P. Miller [Sapindaceae] .18

Solanum atriplicifolium Gill. ex Nees

[Solanaceae].

Solanum elaeagnifolium Cav.[Solanaceae]..5, 10

Solanaceae .19

Sphaeralcea bonariensis Griseb. [Malvaceae] .... $.5,10$

Talguena $[\mathbf{s i c}]$ costata $=$ Talguenea costata $\ldots . .20$

Talguenea costata Miers [Rhamnaceae] ...........20

Tamarix africana Poir. [Tamaricaceae] ........5, 10 Vernonia Schreb. [Asteraceae]. .18

\section{Índice das espécies de Colletes de outras regiões zoogeográficas}

Apis succincta L., 1758 [paleártica] .2

Colletes daviesanus Smith, 1846 [paleártica] ....7

Colletes intermixtus Swenk, 1905 [ neártica]... 18

Colletes nasutus Smith, 1853 [paleártica] .........12

Colletes nudus Robertson, 1898 [neártica] .......15

Colletes prosopidis Cockerell, 1897 [neártica].11

Colletes thoracicus Smith, 1853 [neártica] .......14

\section{Índice dos outros insetos}

Anthrax Scopoli, 1763 [Diptera, Bombyliidae] ... 12

Bombyliidae [Diptera] ....................................... 12

Coelioxys Latreille, 1809 [Hymenoptera, Megachilidae] 3

Copestylum azureum (Philippi, 1865) [Diptera, Syrphidae] ........................................... 8

Diphaglossa Spinola, 1851 [Hymenoptera, Colletidae].......................................... 24

Diptera. 3

Epeolus Latreille, 1802 [Hymenoptera, Anthophoridae] ............................................. 3

Epeolus luctuosus = Isepeolus luctuosus ......... 12

Epeolus rufoclypeus Fox, 1891 14

Epeolus triseriatus $=$ Melectoides triseriatus

Epeoloides septemnotatus $=$ Isepeolus septemnotatus...... 8,15

Hypodynerus chilensis (Spinola, 1851) [Hymenoptera, Vespidae]

Hypodynerus humeralis (Haliday, 1836) [Hymenoptera, Vespidae]

Isepeolus Cockerell, 1907 [Hymenoptera, Anthophoridae]

Isepeolus luctuosus (Spinola, 1851) [Hymenoptera, Anthophoridae]. $4,8,12$

Isepeolus septemnotatus (Spinola, 1851) [Hymenoptera, Anthophoridae] $8,15,20$

Isepeolus triseriatus $=$ Melectoides triseriatus. $4,8,12,15$

Isepeolus viperinus (Holmberg, 1886) [Hymenoptera, Anthophoridae]

Lonchopria zonalis (Reed, 1892) [Hymenoptera, Colletidae]

Melectoides triseriatus (Friese, 1908) [Hymenoptera, Anthophoridae ]......4, 8, 12, 15, 23

Melipona quadrifasciata quadrifasciata Lepeletier, 1836. [Hymenoptera, Apidae] ...... 11

Odynerus Latreille, 1802 [Hymenoptera, Vespidae]

Odynerus humeralis = Hypodynerus humeralis...

Reedapis semicyanea (Spinola, 1851) [Hyme-

noptera, Colletidae]. 8,23

Volucella azurea $=$ Copestylum azureum .

Revta bras. Zool. 19 (1): 1 - 30, 2002 